\title{
Experimental Analysis of Interacting HT22 Plasma Membrane Cholesterol and $\beta$-Amyloid
}

\author{
George Livadiotis ${ }^{*}$, Leila Assas ${ }^{2}$, Maher A. Dayeh ${ }^{1}$, Saber Elaydi ${ }^{3}$, Chloe Phea ${ }^{3}$, \\ James L. Roberts ${ }^{3}$, Yara Samman ${ }^{3}$, Rachel Tchen ${ }^{3}$ \\ ${ }^{1}$ Southwest Research Institute, San Antonio, USA \\ ${ }^{2}$ Department of Mathematics, King Abdul Aziz University, Jeddah, Saudi Arabia \\ ${ }^{3}$ Trinity University, San Antonio, USA \\ Email: ^glivadiotis@swri.edu
}

How to cite this paper: Livadiotis, G., Assas, L., Dayeh, M.A., Elaydi, S., Phea, C., Roberts, J.L., Samman, Y. and Tchen, R. (2017) Experimental Analysis of Interacting HT22 Plasma Membrane Cholesterol and $\beta$-Amyloid. Advances in Alzheimer's Disease, 6, 75-96

https://doi.org/10.4236/aad.2017.64006

Received: November 10, 2017

Accepted: December 22, 2017

Published: December 25, 2017

Copyright (C) 2017 by authors and Scientific Research Publishing Inc. This work is licensed under the Creative Commons Attribution International License (CC BY 4.0).

http://creativecommons.org/licenses/by/4.0/

\begin{abstract}
The peptide $\beta$-Amyloid ( $\beta$-A) is known to be one of the primary factors causing neurodegeneration in the Alzheimer disease. Hence, one would like to know the factors that would increase or decrease the toxicity of $\beta$-Amyloid in the brain. One of the factors that are debated in the literature is cholesterol, where it is not clear if modulating the levels of cholesterol would affect the degree of toxicity of $\beta$-Amyloid on neuron cells in the brain. In order to investigate this problem, data were collected and analyzed for three types of experiments: 1) Correspondence between cholesterol and methyl- $\beta$-cyclodextrin $(\mathrm{M} \beta \mathrm{CD})$ measurements; 2) measurements of the relative fluorescence unit (RFU) with respect to $\mathrm{M} \beta \mathrm{CD}$ concentration (with/without $\beta$-A); and 3) RFU measurements with respect to $\beta$-A concentration (with/without $\mathrm{M} \beta \mathrm{CD}$ ). HT22 hippocampal neurons immortalized with the simian virus SV-40 large $\mathrm{T}$-antigen plasmid vector were used to conduct the experiments. Mito-ID Membrane potential cytotoxicity was used as a measure of mitochondrial potential change. The statistical analysis of the presented experimental results indicates that cholesterol has no statistically significant effect on the degree of toxicity of $\beta$-Amyloid.
\end{abstract}

\section{Keywords}

Alzheimer Disease, $\beta$-Amyloid, Cholesterol

\section{Introduction}

Alzheimer's Disease (AD) is the most prevalent of neurodegenerative diseases among the elderly. Health care costs related to Alzheimer's disease for just this year are estimated to be $\$ 226$ billion. Spending is estimated to increase and reach 
$\$ 1.1$ trillion by 2050. Research to cure Alzheimer's is rather underfunded. Currently, the USA government spends $\sim \$ 1$ billion per year on research-quite less than the cost on health care (source: http://curealz.org). Despite the costs to society and the high prevalence of $\mathrm{AD}$, several decades of research have managed to advance our knowledge on the mechanisms of AD pathology (from its underlying genetics, to the molecular biology and clinical pathology, e.g., [1]). In order to document the growth of the research on $\mathrm{AD}$, we conducted a survey of the Google Scholar for papers related to the AD in their title the last 50 years, from 1967 through mid-2017. Figure 1 demonstrates the results of this survey, where we identified 140,000 papers that refer to AD with their publication rate following an exponential growth since 1985.

The peptide $\beta$-Amyloid ( $\beta$-A) is known to be one of the primary factors causing neurodegeneration in AD. Typically, AD appears with extracellular plaques of $\beta$-A and neurofibrillary tangles in the intracellular environment. There are several theories about the properties of the neuronal plasma membrane that affect the way $\beta$-A interacts with the cell surface. We can observe an increase in oxidative stress as a result of $\beta$-A, but the pathway by which that stress is induced is unclear. There are three main theories about the mechanism behind the ion balance disruption [2] [3] [4]: 1) $\beta$-A inserts into the plasma membrane in its oligomeric form creating ion-specific pores that disrupt ion balance (calcium disruption) [5] [6] ; 2) Aggregation on the surface of the membrane destabilizes the structure and compromises the ability to stop unwanted and non-specific ion flow across the membrane [7] [8]; and 3) $\beta$-A acts as a detergent of the membrane and removes lipid, which subsequently affects membrane structure and fluidity with the same result as the second theory [4]. While the ideas on the mechanisms differ, all three theories agree that the introduction of the peptide to cells leads to oxidative stress.
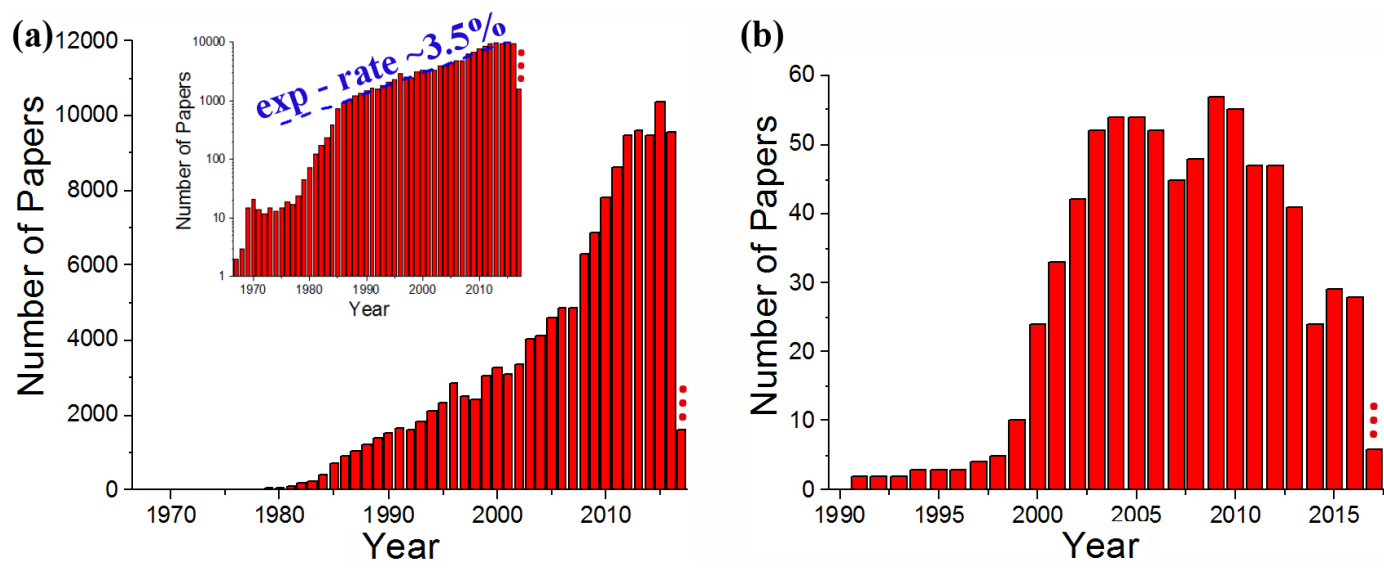

Figure 1. (a) Number of papers cataloged in Google Scholar the last 50 years (1967-2017) that include AD in their title. The fit curve (blue dash) shows the exponential growth of these studies that applies after 1985. In particular, the number of papers $N$ follows the relation $N(t)=N_{1967} \cdot \exp [+\lambda(t-1967)]$ with a rate of $\lambda \cong(3.5 \pm 0.2) \%$ and constant $N_{1967} \cong 344 \pm 10$. (b) The portion of these papers that are related to AD and Cholesterol and include both of these keywords in their title. 
The plasma membrane of a cell is highly functionalized for cell signaling. Many different markers exist on the surface of the cell and interact with extracellular molecules regularly. Cell signaling is not isolated to biomarkers; it also is related to the degree of fluidity in the membrane. Fluidity in the microenvironment can influence cell communication through alteration of diffusion and transfer of cell signaling compounds. This fluidity is determined by the ratio of types of lipids composing the membrane. Increased amounts of cholesterol at physiological temperatures decrease the fluidity of the membrane [9]. This decreased fluidity can have major implications for the ability of extracellular molecules to interact with the cell surface and lateral movement within the plasma membrane. There are specialized areas of the cellular membrane enriched with cholesterol, sphingomyelin, and sphingolipids. These areas are thought to be specialized in intercellular interactions and signaling and are called lipid rafts [10]. The decreased fluidity of these lipid raft regions may increase the chance of interactions between extracellular molecules and the membrane.

Simulations and modeling suggest that $\beta$-A tends to aggregate along the perimeter of these lipid-enriched domains (e.g., see [11]). Disrupting the structure of these specialized membrane rafts could affect the interaction $\beta$-A has with the neuronal membrane. Removing cholesterol would increase the fluidity of the area effectively dissolving the lipid raft. If lipid rafts do play a significant role in $\beta$-A interactions with the neuronal plasma membrane, this removal should affect the level of oxidative stress $\beta$-A is capable of inducing in neurons.

The role of cholesterol in the appearance of $\mathrm{AD}$ was particularly studied during the last two decades (Figure 1(b)). Indeed, the top 100 cited cholesterol papers [12] suggest that the last 10 years have witnessed the advent of studies describing the role of cholesterol in non-cardiovascular diseases such as Alzheimer's (the second reference numbered 76 and 94). As indicated in a review paper by Vance et al. [13], several studies provided conflicting results on the association between plasma cholesterol levels and the development of dementia [14] [15] [16] [17] [18]. The debate focused about how modulating the levels of cholesterol would affect the degree of toxicity of $\beta$-A on the cells. There are three theories about cholesterol levels: 1 ) The presence of cholesterol is beneficial, and its removal would increase levels of oxidative stress [19] [20]; 2) Cholesterol negatively impacts the cell, and its removal would decrease levels of oxidative stress [21]; and 3) Cholesterol does not impact the health of the cell in relation to $\beta-\mathrm{A}$ toxicity.

In order to elaborate on the first theory, removal of cholesterol is possibly negative if we relate it back to the theory that $\beta$-A aggregates along the perimeter of lipid rafts. If cholesterol were removed from the plasma membrane, lipid rafts would first start fractioning into smaller islands. These smaller lipid rafts would have larger total perimeter than the original lipid raft from which it originated. Thus, according to simulations suggesting aggregation along the boundaries of lipid rafts [11], $\beta$-A would have more areas to aggregate upon leading to greater levels of oxidative stress. This also corroborates the finding that astrocytes re- 
place less of the cholesterol in the neuronal membrane as the brain ages [22]. Since turnover of cholesterol remains constant, overall levels of cholesterol in the aging brain decrease. The second theory suggests that less cholesterol would be beneficial. Removing cholesterol creates less lipid rafts, which may reduce the chance of plasma membrane interactions with $\beta$-A. The last theory simply states that cholesterol may not play a crucial role in the toxicity of $\beta$-A to the neuron.

In the literature, there is substantial support for all three theories, which only adds to the ambiguity of $\beta$-A's interactions with the neuronal plasma membrane and the necessity to explore this issue further. Though the literature reports a mixture of results, the mixture may exist due to differences in cell types used as well as the type of assay used to measure oxidative stress, cell viability, or other indicators of $\beta$-A peptides' toxic effect on neuronal cells.

Plasma levels of total and LDL-cholesterol are well known to increase with normal aging both in humans and rodents, while the plasma clearance of LDL has been shown to decrease with age in both humans and rodents (see [23] and refs. therein). While there is a decrease in the amount cholesterol found in the membrane with age (e.g., see [24] [25]), analyses also reported evidence of agedependent increases in cholesterol levels (e.g., see [26] [27]). With the increase in the occurrence of $\mathrm{AD}$ with age, this leads us to believe that these correlational disturbances in cholesterol levels may be in part a cause of AD. In fact, there is an ongoing debate on how modulating the levels of cholesterol would affect the degree of toxicity of $\beta$-Amyloid on neuron cells in the brain.

The purpose of this paper is to investigate whether the cholesterol has effects on the degree of toxicity of $\beta$-Amyloid. In Section 2, we provide information about the materials and reagents used in our experiments. In Section 3, we describe in detail the methods of the three experiments, the procedure, and the collected datasets. Methyl- $\beta$-cyclodextrin $(\mathrm{M} \beta \mathrm{CD})$ is used to deplete cholesterol, while in experiment-1, we find the exact correspondence between them; experiment-2 measures the Relative Fluorescence Unit (RFU) for various values of $\mathrm{M} \beta \mathrm{CD}$ concentration (with/without $\beta$-A); experiment-3 measures RFU for various values of $\beta$-A concentration (with/without $\mathrm{M} \beta \mathrm{CD}$ ). In Section 4 , we provide the results of the three experiments, leading to the conclusion that there are no statistically significant differences of the reaction of $\beta$-A (RFU) with changes in cholesterol content of the plasma membrane. Finally, in Section 5, we discuss the results and briefly summarize the conclusions.

\section{Materials and Reagents}

HT22 hippocampal neurons immortalized with the simian virus SV-40 large T-antigen plasmid vector [28] were used to conduct the experiments. At the present study we use undifferentiated cells to model the pathology of mature neurons. The cells were cultured in 5\% Fetal Bovine Serum (FBS) supplemented DMEM-F12 media containing $1 \%$ penicillin-streptomycin and $1 \%$ L-glutamine at $37^{\circ} \mathrm{C}$ and $5 \% \mathrm{CO}_{2}$. A 96-well Costar black clear-bottom plate was used for the 
assays. We used Aggresure ( 1 - 42) $\beta$-amyloid $(\beta$-A) from Anaspec to use in our model of $\mathrm{AD}$. Cholesterol was removed using methyl- $\beta$-cyclodextrin $(\mathrm{M} \beta \mathrm{CD})$, a cyclic molecule with a structure suitable to extract a cholesterol molecule from the surface of plasma membrane; the amounts removed were quantified using the Amplex Red cholesterol quantification kit. Mito-ID Membrane potential cyto toxicity kit was used as a measure of mitochondrial potential change induced in our assays (see details of the product in: Enzo Life Science, http://www.enzolifesciences.com).

We modified the protocol and reagent mixture with no effect on the efficacy

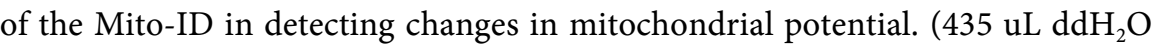
+ $50 \mathrm{uL} 10 \mathrm{X}$ buffer + $10 \mathrm{uL} 50 \mathrm{X}$ buffer + $5 \mathrm{uL}$ Mito-ID MP Detection Reagent; $50 \mathrm{uL}$ into each well for a total volume of $100 \mathrm{uL}$; diluted in $50 \mathrm{uL}$ of $0 \%$ FBS DMEM-F12; the dye was allowed to incubate for $90 \mathrm{~min}$ before taking the baseline reading instead of the suggested incubation time of $30 \mathrm{~min}$ ). A fluorescence microplate reader from Molecular Devices was used to collect readings (excitation $485 \mathrm{~nm}$, emission at 530 and $590 \mathrm{~nm}$ ).

\section{Methods}

\subsection{Description of the Procedure}

Cells were plated into a 96-well clear-bottomed black tissue culture plate about 24 hours before the start of assay to allow the cells to adhere completely to the tissue culture plate. At the start of the assay, cells were verified to be at $70-80 \%$ confluency. The media was aspirated off and cells were rinsed with $100 \mathrm{uL}$ of $0 \%$ FBS DMEM-F12 media supplemented with $1 \%$ pen-strep and $1 \%$ Glutamax. Cells received $100 \mu \mathrm{L}$ of $0 \%$ FBS DMEM-F12 media solutions of 0 to $2.5 \mathrm{mM}$ of $\mathrm{M} \beta \mathrm{CD}$. The cells were placed back in the incubator for 60 minutes at $37^{\circ} \mathrm{C}$. After 60 minutes, the media containing the $\mathrm{M} \beta \mathrm{CD}$ was removed and $50 \mu \mathrm{L}$ of $0 \% \mathrm{FBS}$ DMEM-F12 1\% p-s and 1\% L-Gln was added. $50 \mu \mathrm{L}$ of our Mito-ID mixture was added and the cells were allowed incubate for 90 minutes. After $90 \mathrm{~min}$, the baseline reading was taken with an excitation of $485 \mathrm{~nm}$ and emission read at 530 and $590 \mathrm{~nm}$. Within $5 \mathrm{~min}$ of the baseline reading, $\beta$-A $(1-42)$ peptide was added in varying amounts to the cells. The first reading was taken 15 minutes after the final addition of the $\beta$-A. For the first 60 minutes, readings were taken every 15 minutes. Thereafter, readings were taken every 30 minutes for up to 8 hours post-baseline reading.

The relative fluorescence unit (RFU) represents the mitochondrial activation. Mito-ID is a fluorescence dye that measures the fluctuation of the mitochondrial membrane potential. It uses a cationic dual emission dye that fluoresces green $(530 \mathrm{~nm})$ when in its monomer form, and orange $(590 \mathrm{~nm})$ when it forms J-aggregates due to increasing concentrations within the mitochondria (e.g., [29]). When the cell is healthy, the Mito-ID fluoresces green since the cation is in the cytosol; however, when the cell undergoes oxidative stress, its mitochondria takes up the Mito-ID which in turn aggregates it and shifts the fluorescence to orange. 
In this paper, we will use RFU to measure the cellular variability of neuron cells. The data was normalized to $100 \%$, thus the baseline of a healthy neuron started at $100 \%$ RFU units. The $\operatorname{RFU}(t)$ response values are normalized to the initial $\mathrm{RFU}(t=0)$, i.e.,

$$
\text { Normalized } \operatorname{RFU}(t)=(100 \%) \cdot \operatorname{RFU}(t) / \operatorname{RFU}(t=0) .
$$

Consequently, an increase in relative fluorescence unit represents an increase in mitochondrial activation.

\subsection{Collected Datasets}

We operated three types of experiments: 1) Cholesterol and $\mathrm{M} \beta \mathrm{CD}$ measurements; 2) RFU measurements with respect to $M \beta C D$ concentration (with/without $\beta$-A); and 3) RFU measurements with respect to $\beta$-A concentration (with/without $\mathrm{M} \beta \mathrm{CD})$.

All experiments had been repeated multiple times in order to estimate the sample average values and uncertainties. In experiment (1), for each $\mathrm{M} \beta \mathrm{CD}$ concentration, we have measured RFU and Cholesterol six times. In experiments (2) and (3), at each $t[\mathrm{~min}]=0,15,30,45,60,90,120,150,180,210,250,270$ (or, 1060 for (b)), and for all the concentrations of $\mathrm{M} \beta \mathrm{CD}$ and $\beta$-A, we have measured RFU three times.

- In experiment-1, we first produce the reference curve between Cholesterol $[\mu \mathrm{g}](\mathrm{x})$ and normalized RFU (y), that is, $\mathrm{y}=41,224 \mathrm{x}+559.23$. The averaged values/standard deviations of $\mathrm{M} \beta \mathrm{CD}[\mu \mathrm{M}]$ and normalized RFU are shown in Table 1. Then, using the produced reference curve, we extract the values of Cholesterol $[\mu \mathrm{g}]$, shown also in Table 1.

- In experiment-2, the averaged values and standard deviations of the normalized $\mathrm{RFU}(\mathrm{t}$ ) (for all $\mathrm{t}$ ), for $\mathrm{M} \beta \mathrm{CD}[\mu \mathrm{M}]: 0,0.5,1,1.5,2,2.5$, and both the cases of $0 \beta$-A and $2.5 \beta$-A, are shown in Table 2 .

- In experiment-3, the averaged values and standard deviations of the normalized RFU(t) (for all $\mathrm{t}$ ), for $\beta$-A: $0,1,2,2.5,3,4,5,7,10,15$, and the three cases of $\mathrm{M} \beta \mathrm{CD}[\mu \mathrm{M}]: 0,1,2.5 \beta$-A, are shown in Table 3.

\section{Results}

\subsection{Experiment-1: Cholesterol Assay}

Using Table 1, we plot Cholesterol $[\mu \mathrm{g}]$ with respect to $\mathrm{M} \beta \mathrm{CD}[\mu \mathrm{M}]$. The Amp-

Table 1. Measurements of cholesterol and normalized RFU for each $\mathrm{M} \beta \mathrm{CD}$.

\begin{tabular}{ccc}
\hline $\mathrm{M} \beta \mathrm{CD}[\mu \mathrm{M}]$ & RFU Normalized & Cholesterol $[\mu \mathrm{g}]$ \\
\hline 0 & $11,160 \pm 290$ & $0.260 \pm 0.007$ \\
0.5 & $10,000 \pm 300$ & $0.227 \pm 0.008$ \\
1 & $6800 \pm 300$ & $0.150 \pm 0.008$ \\
1.5 & $4500 \pm 600$ & $0.090 \pm 0.014$ \\
2 & $3500 \pm 600$ & $0.070 \pm 0.015$ \\
2.5 & $3200 \pm 700$ & $0.060 \pm 0.017$ \\
\hline
\end{tabular}


Table 2. Measurements of the normalized RFU for each $\mathrm{M} \beta \mathrm{CD}$, with/without $\beta$-A, at each time $t$.

\begin{tabular}{|c|c|c|c|c|}
\hline \multicolumn{3}{|c|}{$t[\min ]=15$} & \multicolumn{2}{|c|}{$t[\mathrm{~min}]=30$} \\
\hline $\mathbf{M} \beta \mathrm{CD}[\mu \mathrm{M}]$ & $0 \beta$-A & $2.5 \beta-\mathrm{A}$ & $0 \beta-\mathrm{A}$ & $2.5 \beta-\mathrm{A}$ \\
\hline 0 & $111.5 \pm 0.8$ & $150 \pm 30$ & $118.2 \pm 1.5$ & $150 \pm 30$ \\
\hline 0.5 & $110.5 \pm 2.4$ & $195 \pm 14$ & $114.8 \pm 2.0$ & $206 \pm 9$ \\
\hline 1 & $112 \pm 4$ & $174 \pm 16$ & $115 \pm 6$ & $175 \pm 14$ \\
\hline 1.5 & $111.4 \pm 1.0$ & $182 \pm 4$ & $116.5 \pm 1.0$ & $192 \pm 7$ \\
\hline 2 & $111.7 \pm 1.0$ & $176 \pm 22$ & $117.7 \pm 2.2$ & $182 \pm 26$ \\
\hline 2.5 & $119 \pm 8$ & $168 \pm 15$ & $122 \pm 8$ & $178 \pm 20$ \\
\hline \multicolumn{3}{|c|}{$t[\min ]=45$} & \multicolumn{2}{|c|}{$t[\min ]=60$} \\
\hline $\mathbf{M} \beta \mathrm{CD}[\mu \mathrm{M}]$ & $0 \beta-\mathrm{A}$ & $2.5 \beta-\mathrm{A}$ & $0 \beta-\mathrm{A}$ & $2.5 \beta-\mathrm{A}$ \\
\hline 0 & $121.8 \pm 1.1$ & $160 \pm 30$ & $129.1 \pm 1.6$ & $170 \pm 30$ \\
\hline 0.5 & $119.9 \pm 2.4$ & $220 \pm 9$ & $125.0 \pm 2.9$ & $234 \pm 12$ \\
\hline 1 & $123 \pm 6$ & $182 \pm 16$ & $128 \pm 8$ & $194 \pm 20$ \\
\hline 1.5 & $123.1 \pm 0.7$ & $199 \pm 9$ & $129.3 \pm 1.4$ & $209 \pm 10$ \\
\hline 2 & $122.3 \pm 2.5$ & $183 \pm 23$ & $129 \pm 4$ & $200 \pm 30$ \\
\hline 2.5 & $131 \pm 8$ & $192 \pm 26$ & $138 \pm 9$ & $200 \pm 30$ \\
\hline \multicolumn{3}{|c|}{$t[\min ]=90$} & \multicolumn{2}{|c|}{$t[\min ]=120$} \\
\hline $\mathbf{M} \beta \mathrm{CD}[\mu \mathrm{M}]$ & $0 \beta$-A & $2.5 \beta-\mathrm{A}$ & $0 \beta$-A & $2.5 \beta-\mathrm{A}$ \\
\hline 0 & $121.1 \pm 1.8$ & $150 \pm 30$ & $112.5 \pm 2.5$ & $141 \pm 28$ \\
\hline 0.5 & $120.4 \pm 2.4$ & $217 \pm 10$ & $108.1 \pm 0.9$ & $203 \pm 9$ \\
\hline 1 & $122 \pm 7$ & $187 \pm 16$ & $112 \pm 5$ & $168 \pm 16$ \\
\hline 1.5 & $124 \pm 4$ & $203 \pm 7$ & $112 \pm 4$ & $185 \pm 6$ \\
\hline 2 & $122 \pm 4$ & $217 \pm 23$ & $108 \pm 4$ & $167 \pm 18$ \\
\hline 2.5 & $131 \pm 8$ & $196 \pm 20$ & $108 \pm 5$ & $175 \pm 23$ \\
\hline \multicolumn{3}{|c|}{$t[\min ]=150$} & \multicolumn{2}{|c|}{$t[\min ]=180$} \\
\hline $\mathbf{M} \beta \mathrm{CD}[\mu \mathrm{M}]$ & $0 \beta-A$ & $2.5 \beta-\mathrm{A}$ & $0 \beta-A$ & $2.5 \beta-\mathrm{A}$ \\
\hline 0 & $111.1 \pm 2.7$ & $170 \pm 30$ & $108 \pm 3$ & $143 \pm 23$ \\
\hline 0.5 & $105.5 \pm 1.4$ & $193 \pm 8$ & $101.8 \pm 0.8$ & $183 \pm 7$ \\
\hline 1 & $108 \pm 5$ & $162 \pm 15$ & $103.5 \pm 3.2$ & $158 \pm 12$ \\
\hline 1.5 & $109 \pm 3$ & $177 \pm 7$ & $104 \pm 4$ & $167 \pm 8$ \\
\hline 2 & $104 \pm 3$ & $165 \pm 24$ & $99 \pm 4$ & $155 \pm 19$ \\
\hline 2.5 & $107 \pm 8$ & $168 \pm 22$ & $101 \pm 6$ & $158 \pm 19$ \\
\hline \multicolumn{3}{|c|}{$t[\min ]=210$} & \multicolumn{2}{|c|}{$t[\min ]=240$} \\
\hline $\mathrm{M} \beta \mathrm{CD}[\mu \mathrm{M}]$ & $0 \beta$-A & $2.5 \beta-\mathrm{A}$ & $0 \beta-A$ & $2.5 \beta-\mathrm{A}$ \\
\hline 0 & $107 \pm 4$ & $140 \pm 23$ & $116 \pm 4$ & $155 \pm 26$ \\
\hline 0.5 & $99.5 \pm 1.5$ & $178 \pm 8$ & $107.9 \pm 2.0$ & $197 \pm 8$ \\
\hline
\end{tabular}




\section{Continued}

\begin{tabular}{ccccc}
\hline 1 & $101 \pm 5$ & $154 \pm 12$ & $110 \pm 7$ & $164 \pm 16$ \\
1.5 & $101 \pm 3$ & $163 \pm 5$ & $111 \pm 3$ & $177 \pm 7$ \\
2 & $96.2 \pm 2.9$ & $159 \pm 24$ & $107 \pm 4$ & $176 \pm 34$ \\
2.5 & $102 \pm 8$ & $157 \pm 20$ & $116 \pm 9$ & $169 \pm 25$ \\
\hline $\mathbf{M} \beta \mathrm{CD}[\mu \mathrm{M}]$ & 0 ( $\beta$ - $\mathrm{min}]=1060$ & & & \\
0 & $97.6 \pm 1.0$ & $127 \pm 20$ & & \\
0.5 & $85.4 \pm 2.4$ & $169 \pm 9$ & & \\
1 & $84 \pm 9$ & $122 \pm 19$ & & \\
1.5 & $95 \pm 4$ & $143 \pm 7$ & & \\
2 & $83 \pm 4$ & $120 \pm 30$ & & \\
2.5 & $90 \pm 6$ & $120 \pm 30$ & & \\
\hline
\end{tabular}

Table 3. Measurements of normalized RFU for each $\beta$-A, with/without $\mathrm{M} \beta \mathrm{CD}$, at each time $t$.

\begin{tabular}{|c|c|c|c|c|c|c|}
\hline \multicolumn{4}{|c|}{$t[\min ]=15$} & \multicolumn{3}{|c|}{$t[\mathrm{~min}]=30$} \\
\hline$\beta-\mathrm{A}$ & $0 \mathrm{M} \beta \mathrm{CD}$ & $1 \mathrm{M} \beta \mathrm{CD}$ & $2.5 \mathrm{M} \beta \mathrm{CD}$ & $0 \mathrm{M} \beta \mathrm{CD}$ & $1 \mathrm{M} \beta \mathrm{CD}$ & $2.5 \mathrm{M} \beta \mathrm{CD}$ \\
\hline 0 & $108.0 \pm 1.9$ & $107.8 \pm 1.5$ & $105.7 \pm 1.3$ & $111.1 \pm 1.5$ & $111.5 \pm 1.2$ & $107.7 \pm 2.7$ \\
\hline 1 & $108.4 \pm 2.0$ & $106.1 \pm 2.7$ & $110 \pm 6$ & $111.4 \pm 1.5$ & $110.7 \pm 2.4$ & $114 \pm 7$ \\
\hline 2 & $133 \pm 15$ & $170 \pm 50$ & $180 \pm 30$ & $133 \pm 15$ & $170 \pm 50$ & $180 \pm 30$ \\
\hline 2.5 & $112 \pm 5$ & $111 \pm 6$ & $122 \pm 16$ & $122.6 \pm 2.3$ & $117 \pm 9$ & $125 \pm 16$ \\
\hline 3 & $185 \pm 24$ & $200 \pm 60$ & $190 \pm 50$ & $187 \pm 27$ & $200 \pm 60$ & $200 \pm 60$ \\
\hline 4 & $212 \pm 34$ & $190 \pm 50$ & $210 \pm 80$ & $208 \pm 29$ & $210 \pm 70$ & $210 \pm 70$ \\
\hline 5 & $201 \pm 30$ & $200 \pm 50$ & $200 \pm 50$ & $194 \pm 25$ & $200 \pm 40$ & $200 \pm 50$ \\
\hline 7 & $166 \pm 25$ & $157 \pm 21$ & $144 \pm 16$ & $172 \pm 24$ & $172 \pm 22$ & $162 \pm 27$ \\
\hline 10 & $410 \pm 120$ & $450 \pm 150$ & $340 \pm 110$ & $420 \pm 120$ & $450 \pm 140$ & $350 \pm 100$ \\
\hline 15 & $310 \pm 30$ & $250 \pm 50$ & $202 \pm 16$ & $330 \pm 30$ & $260 \pm 50$ & $208 \pm 16$ \\
\hline \multicolumn{4}{|c|}{$t[\min ]=45$} & \multicolumn{3}{|c|}{$t[\min ]=60$} \\
\hline$\beta-A$ & $0 \mathrm{M} \beta \mathrm{CD}$ & $1 \mathrm{M} \beta \mathrm{CD}$ & $2.5 \mathrm{M} \beta \mathrm{CD}$ & $0 \mathrm{M} \beta \mathrm{CD}$ & $1 \mathrm{M} \beta \mathrm{CD}$ & $2.5 \mathrm{M} \beta \mathrm{CD}$ \\
\hline 0 & $112.5 \pm 1.6$ & $113.4 \pm 2.3$ & $111.0 \pm 2.9$ & $117 \pm 3$ & $117.2 \pm 1.6$ & $116 \pm 5$ \\
\hline 1 & $113.5 \pm 2.4$ & $117 \pm 6$ & $117 \pm 8$ & $117.0 \pm 2.6$ & $121 \pm 6$ & $121 \pm 10$ \\
\hline 2 & $140 \pm 19$ & $170 \pm 50$ & $173 \pm 30$ & $144 \pm 18$ & $180 \pm 50$ & $180 \pm 29$ \\
\hline 2.5 & $123 \pm 3$ & $118 \pm 10$ & $127.1 \pm 17$ & $124.3 \pm 1.8$ & $118 \pm 11$ & $129 \pm 19$ \\
\hline 3 & $210 \pm 40$ & $200 \pm 60$ & $190 \pm 50$ & $220 \pm 50$ & $210 \pm 70$ & $210 \pm 50$ \\
\hline 4 & $209 \pm 27$ & $220 \pm 70$ & $210 \pm 70$ & $210 \pm 30$ & $230 \pm 80$ & $220 \pm 70$ \\
\hline 5 & $194 \pm 24$ & $210 \pm 40$ & $200 \pm 40$ & $198 \pm 27$ & $210 \pm 50$ & $200 \pm 50$ \\
\hline 7 & $180 \pm 30$ & $176 \pm 25$ & $163 \pm 29$ & $180 \pm 30$ & $181 \pm 29$ & $170 \pm 30$ \\
\hline 10 & $430 \pm 120$ & $460 \pm 140$ & $360 \pm 110$ & $450 \pm 130$ & $470 \pm 150$ & $370 \pm 120$ \\
\hline
\end{tabular}




\section{Continued}

\begin{tabular}{|c|c|c|c|c|c|c|}
\hline 15 & $325 \pm 37$ & $260 \pm 50$ & $212 \pm 14$ & $329 \pm 29$ & $270 \pm 50$ & $214 \pm 17$ \\
\hline \multicolumn{4}{|c|}{$t[\mathrm{~min}]=90$} & \multicolumn{3}{|c|}{$t[\min ]=120$} \\
\hline$\beta-\mathrm{A}$ & $0 \mathrm{M} \beta \mathrm{CD}$ & $1 \mathrm{M} \beta \mathrm{CD}$ & $2.5 \mathrm{M} \beta \mathrm{CD}$ & $0 \mathrm{M} \beta \mathrm{CD}$ & $1 \mathrm{M} \beta \mathrm{CD}$ & $2.5 \mathrm{M} \beta \mathrm{CD}$ \\
\hline 0 & $107.2 \pm 2.5$ & $105.4 \pm 1.7$ & $101.5 \pm 2.7$ & $103.2 \pm 2.9$ & $100.3 \pm 2.5$ & $97 \pm 4$ \\
\hline 1 & $107.7 \pm 2.9$ & $110 \pm 7$ & $110 \pm 6$ & $105 \pm 3$ & $104 \pm 7$ & $103 \pm 4$ \\
\hline 2 & $132 \pm 14$ & $160 \pm 50$ & $160 \pm 30$ & $125 \pm 12$ & $160 \pm 50$ & $150 \pm 40$ \\
\hline 2.5 & $120.7 \pm 2.7$ & $111 \pm 14$ & $121 \pm 23$ & $122 \pm 10$ & $106 \pm 15$ & $116 \pm 25$ \\
\hline 3 & $200 \pm 50$ & $190 \pm 60$ & $190 \pm 50$ & $200 \pm 50$ & $180 \pm 60$ & $180 \pm 60$ \\
\hline 4 & $190 \pm 40$ & $210 \pm 70$ & $200 \pm 70$ & $180 \pm 40$ & $200 \pm 70$ & $190 \pm 70$ \\
\hline 5 & $184 \pm 23$ & $200 \pm 40$ & $190 \pm 40$ & $179 \pm 23$ & $190 \pm 40$ & $180 \pm 40$ \\
\hline 7 & $170 \pm 27$ & $170 \pm 30$ & $160 \pm 30$ & $170 \pm 29$ & $160 \pm 30$ & $150 \pm 40$ \\
\hline 10 & $420 \pm 130$ & $450 \pm 150$ & $350 \pm 120$ & $410 \pm 130$ & $430 \pm 150$ & $340.5 \pm 120$ \\
\hline 15 & $309 \pm 23$ & $250 \pm 60$ & $205 \pm 23$ & $298 \pm 12$ & $240 \pm 70$ & $190 \pm 30$ \\
\hline \multicolumn{4}{|c|}{$t[\min ]=150$} & \multicolumn{3}{|c|}{$t[\min ]=180$} \\
\hline$\beta-\mathrm{A}$ & $0 \mathrm{M} \beta \mathrm{CD}$ & $1 \mathrm{M} \beta \mathrm{CD}$ & $2.5 \mathrm{M} \beta \mathrm{CD}$ & $0 \mathrm{M} \beta \mathrm{CD}$ & $1 \mathrm{M} \beta \mathrm{CD}$ & $2.5 \mathrm{M} \beta \mathrm{CD}$ \\
\hline 0 & $102 \pm 3$ & $98.9 \pm 3$ & $94 \pm 5$ & $111 \pm 4$ & $106 \pm 3$ & $100 \pm 4$ \\
\hline 1 & $103 \pm 3$ & $105.0 \pm 8$ & $100 \pm 3$ & $110 \pm 4$ & $114 \pm 11$ & $105 \pm 5$ \\
\hline 2 & $120 \pm 10$ & $153.8 \pm 50$ & $150 \pm 40$ & $132 \pm 14$ & $160 \pm 50$ & $150 \pm 40$ \\
\hline 2.5 & $119 \pm 8$ & $104.1 \pm 14$ & $115 \pm 24$ & $123 \pm 11$ & $107 \pm 14$ & $117 \pm 25$ \\
\hline 3 & $190 \pm 50$ & $176.7 \pm 70$ & $170 \pm 60$ & $200 \pm 50$ & $190 \pm 70$ & $180 \pm 60$ \\
\hline 4 & $180 \pm 40$ & $196.5 \pm 60$ & $190 \pm 70$ & $190 \pm 30$ & $210 \pm 70$ & $200 \pm 70$ \\
\hline 5 & $176 \pm 24$ & $184.4 \pm 50$ & $180 \pm 40$ & $184 \pm 25$ & $190 \pm 50$ & $180 \pm 50$ \\
\hline 7 & $168 \pm 29$ & $160.0 \pm 40$ & $150 \pm 40$ & $170 \pm 30$ & $160 \pm 40$ & $150 \pm 40$ \\
\hline 10 & $400 \pm 140$ & $430.8 \pm 150$ & $350 \pm 140$ & $420 \pm 140$ & $450 \pm 160$ & $370 \pm 140$ \\
\hline 15 & $291 \pm 9$ & $233.6 \pm 80$ & $187.8 \pm 30$ & $302 \pm 14$ & $240 \pm 80$ & $190 \pm 40$ \\
\hline \multicolumn{4}{|c|}{$t[\mathrm{~min}]=210$} & \multicolumn{3}{|c|}{$t[\min ]=240$} \\
\hline$\beta-\mathrm{A}$ & $0 \mathrm{M} \beta \mathrm{CD}$ & $1 \mathrm{M} \beta \mathrm{CD}$ & $2.5 \mathrm{M} \beta \mathrm{CD}$ & $0 \mathrm{M} \beta \mathrm{CD}$ & $1 \mathrm{M} \beta \mathrm{CD}$ & $2.5 \mathrm{M} \beta \mathrm{CD}$ \\
\hline 0 & $112 \pm 3$ & $107.9 \pm 2.9$ & $101.1 \pm 2.7$ & $114 \pm 7$ & $109 \pm 6$ & $101 \pm 4$ \\
\hline 1 & $110 \pm 5$ & $108 \pm 6$ & $106 \pm 6$ & $109 \pm 6$ & $108 \pm 9$ & $107 \pm 7$ \\
\hline 2 & $130 \pm 20$ & $160 \pm 40$ & $150 \pm 30$ & $136 \pm 18$ & $160 \pm 50$ & $160 \pm 40$ \\
\hline 2.5 & $125 \pm 10$ & $108 \pm 16$ & $119 \pm 26$ & $121 \pm 13$ & $105 \pm 17$ & $118 \pm 25$ \\
\hline 3 & $197 \pm 40$ & $180 \pm 60$ & $180 \pm 50$ & $205.3 \pm 40$ & $190 \pm 60$ & $190 \pm 60$ \\
\hline 4 & $184 \pm 29$ & $200 \pm 70$ & $190 \pm 70$ & $192 \pm 28$ & $210 \pm 70$ & $200 \pm 70$ \\
\hline 5 & $182 \pm 22$ & $190 \pm 40$ & $180 \pm 40$ & $181 \pm 25$ & $190 \pm 50$ & $180 \pm 40$ \\
\hline 7 & $177 \pm 30$ & $160 \pm 50$ & $150 \pm 50$ & $170 \pm 30$ & $160 \pm 50$ & $150 \pm 50$ \\
\hline 10 & $420 \pm 120$ & $440 \pm 140$ & $350 \pm 130$ & $430 \pm 130$ & $450 \pm 150$ & $370 \pm 140$ \\
\hline 15 & $309 \pm 17$ & $250 \pm 80$ & $190 \pm 40$ & $301 \pm 7$ & $240 \pm 90$ & $190 \pm 40$ \\
\hline
\end{tabular}




\section{Continued}

\begin{tabular}{cccc}
\hline \multicolumn{3}{c}{$t[\mathrm{~min}]=270$} \\
$\beta-\mathrm{A}$ & $0 \mathrm{M} \beta \mathrm{CD}$ & $1 \mathrm{M} \beta \mathrm{CD}$ & $2.5 \mathrm{M} \beta \mathrm{CD}$ \\
0 & $111 \pm 7$ & $107 \pm 7$ & $98 \pm 5$ \\
1 & $106 \pm 6$ & $106 \pm 10$ & $104 \pm 8$ \\
2 & $132 \pm 14$ & $160 \pm 50$ & $160 \pm 40$ \\
2.5 & $115 \pm 15$ & $101 \pm 19$ & $119 \pm 22$ \\
3 & $220 \pm 30$ & $190 \pm 70$ & $180 \pm 60$ \\
4 & $190 \pm 30$ & $210 \pm 80$ & $200 \pm 80$ \\
5 & $180 \pm 27$ & $190 \pm 50$ & $180 \pm 50$ \\
7 & $170 \pm 30$ & $160 \pm 50$ & $140 \pm 50$ \\
10 & $430 \pm 140$ & $460 \pm 160$ & $370 \pm 150$ \\
15 & $291.3 \pm 1.5$ & $240 \pm 90$ & $190 \pm 50$ \\
\hline
\end{tabular}

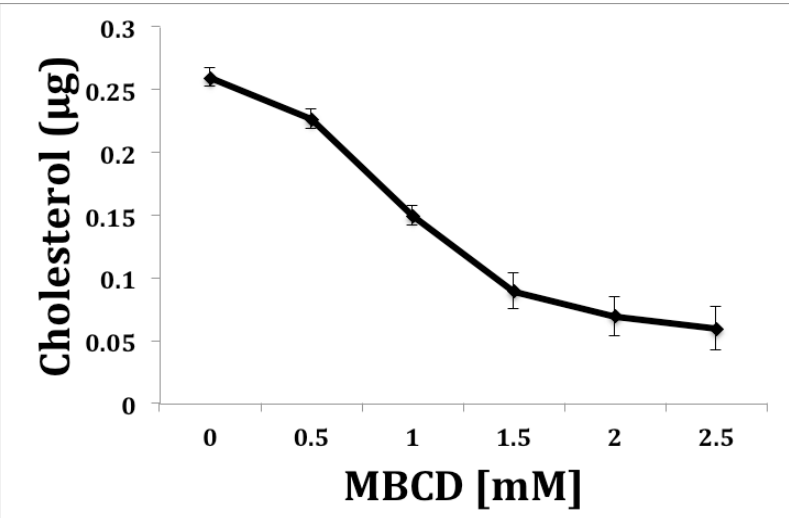

Figure 2. Cholesterol concentration $[\mu \mathrm{g}]$ vs the concentration of $\mathrm{M} \beta \mathrm{CD}$ in $[\mathrm{mM}] . \mathrm{M} \beta \mathrm{CD}$ was added in concentrations from 0 to 2.5 to HT22 hippocampal cells. Amplex red assay fluoresces with the amount of Cholesterol available.

lex Red cholesterol assay revealed a dose dependent decrease in cholesterol according to the $\mathrm{M} \beta \mathrm{CD}$ concentration, as shown in Figure 2. A $2.5 \mathrm{mM}$ treatment of $\mathrm{M} \beta \mathrm{CD}$ resulted in the extraction of around $75 \%$ of the neuron's cholesterol. Between 1 and $1.5 \mathrm{mM}$ of $\mathrm{M} \beta \mathrm{CD}$ resulted in approximately $50 \%$ cholesterol extraction. At higher concentrations of $\mathrm{M} \beta \mathrm{CD}$ the cells died.

\subsection{Experiment-2: Variation of $\mathrm{M} \beta \mathrm{CD}$}

Figure 3 shows the normalized RFU plotted with respect to $\mathrm{M} \beta \mathrm{CD}$, for (a) $0 \beta$-A and (b) $2.5 \beta$-A (data shown in Table 2).

In the statistical tests that will follow, we use the RFU normalized values in order to determine if the statistical population is invariant with respect to changes of the $\mathrm{M} \beta \mathrm{CD}$ concentration. In order to perform this examination, statistical tests are applied to the following null-hypothesis: 

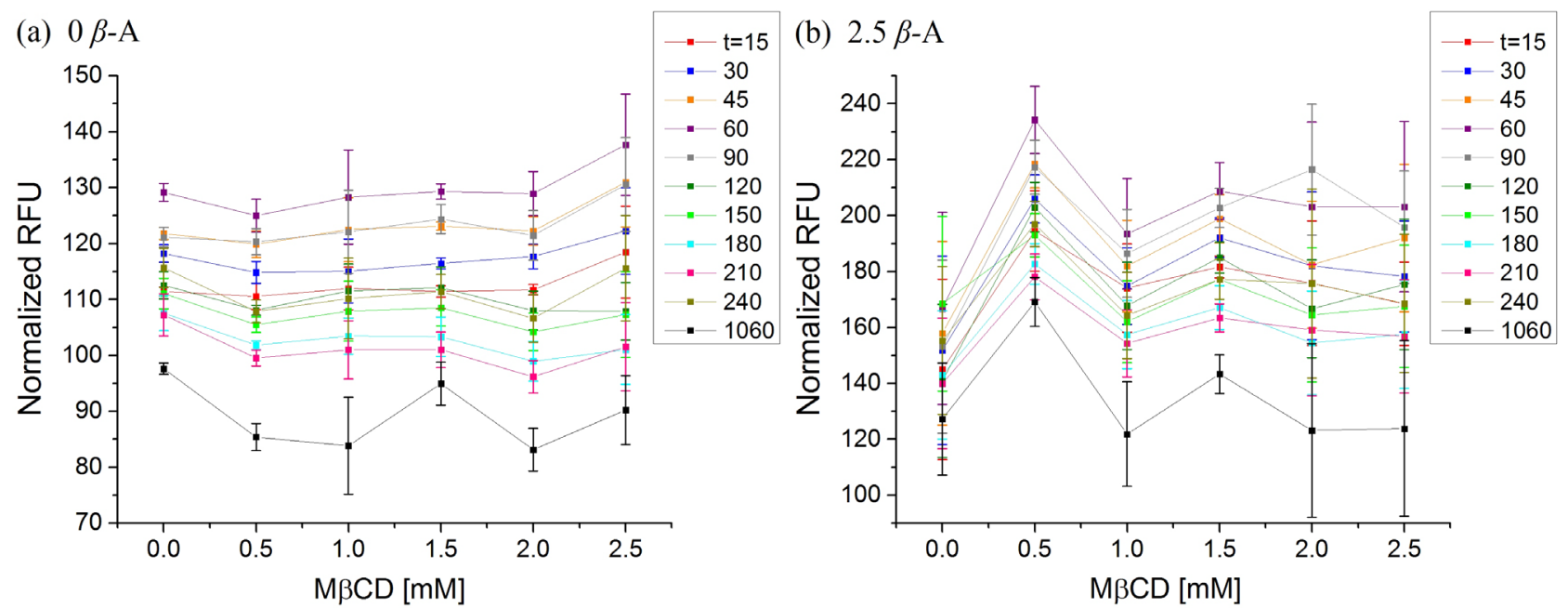

Figure 3. Normalized RFU values plotted with respect to $\mathrm{M} \beta \mathrm{CD}[\mathrm{mM}]$ for times $t[\mathrm{~min}]=0,15,30,45,60,90,120,150,180,210$, 250,1060 , for (a) $0 \beta$-A and (b) $2.5 \beta$-A.

\section{- $\mathrm{H}_{0}$ : The response $\mathrm{RFU}$ is invariant under variations of $M \beta C D$.}

Next, we apply a standard statistical analysis (e.g., [30] [31]) to show whether the hypothesis can be accepted or rejected. In particular, the null-hypothesis is tested using a statistical method to rate the goodness of the involved fit. A good fit of data to a constant favors $\mathrm{H}_{0}$. Three statistical methods are used to rate the goodness of the fitting, i) the "reduced chi-square", ii) the " $p$-value of the extremes", and iii) the "T-value", that is, a transformed $p$-value; (similar applications of the statistical tests can be found in examples of different disciplines, e.g., [32] [33] [34] [35] [36]).

- For the chi-square method, the goodness of a fit is estimated by the reduced chi-square value, $\chi_{\text {red }}^{2}=\frac{1}{M} \chi_{\text {est }}^{2}$, where $M$ are the degrees of freedom. The meaning of $\chi_{\text {red }}^{2}$ is the portion of $\chi^{2}$ that corresponds to each of the degrees of freedom, and this has to be $\sim 1$ for a good fit. Therefore, a fit is characterized as "good" when $\chi_{\text {red }}^{2} \sim 1$, otherwise there is an overestimation, $\left(\chi_{\text {red }}^{2}<1\right)$, or underestimation, $\left(\chi_{\text {red }}^{2}>1\right)$, of the errors.

For the $p$-value method, the goodness of the fit is evaluated by comparing the estimated chi-square value, $\chi_{\text {est }}^{2}$, and the chi-square distribution,

$$
P\left(\chi^{2} ; M\right)=\left[2^{\frac{M}{2}} \Gamma\left(\frac{M}{2}\right)\right]^{-1} e^{-\frac{1}{2} \chi^{2}}\left(\chi^{2}\right)^{\frac{M}{2}-1},
$$

that is, the distribution of all the possible $\chi^{2}$ values (parameterized by the degrees of freedom $M$ ). The likelihood of having a $\chi^{2}$ value, equal to or larger than the estimated value $\chi_{\text {est }}^{2}$, is given by the complementary cumulative distribution. The probability of taking a result $\chi^{2}$, larger than the estimated value $\chi_{\text {est }}^{2}$, defines the $p$-value that equals

$$
P\left(\chi_{\text {est }}^{2} \leq \chi^{2}<\infty\right)=\int_{\chi_{\text {est }}^{2}}^{\infty} P\left(\chi^{2} ; M\right) d \chi^{2} .
$$

The larger the $p$-value, the better the fit: a $p$-value larger than 0.5 corresponds 
to $\chi_{\text {est }}^{2}<M$ or $\chi_{\text {red }}^{2}<1$. Larger $p$-values, up to $p=1$, correspond to smaller chi-squares, down to $\chi_{\text {red }}^{2} \sim 0$. Thus, an increasing $p$-value above the threshold of 0.5 cannot lead to a better fitting. Rather, it leads to a worse fit, similar to a decreasing $\chi_{\text {red }}^{2}<1$. For this reason, the " $p$-value of the extremes" is used. According to this method, the probability of taking a result $\chi^{2}$, that is more extreme than the observed value $\chi_{\text {est }}^{2}$, defines the $p$-value that equals the minimum between the two probabilities, $P\left(0 \leq \chi^{2} \leq \chi_{\text {est }}^{2}\right)$ and its complementary, $P\left(\chi_{\text {est }}^{2} \leq \chi^{2}<\infty\right)$. A null hypothesis associated with a $p$-value smaller than the significance level of $\sim 0.05$ is typically rejected.

As shown in Table 4, all the estimated $p$-values were above the $5 \%$ confident level, which is required for the acceptance of the hypothesis. We also use the T-value, a modified $p$-value, in order to characterize the likelihood in more detail. This is defined by

$$
(\mathrm{T}+1) / 2=(2 p)^{\log 2} \text {. }
$$

Figure 4 plots the histograms of all the estimated (a) $p$ - and (b) T-values, for the cases of $\beta$-A $=0$ (upper panels), $\beta$-A $=2.5$ (middle panels), or all together (lower panels). We observe that all the cases of $\beta$-A $=2.5$ have $p$-value larger than 0.05 , indicating that the hypothesis of invariant RFU under variations of $\mathrm{M} \beta \mathrm{CD}$ can be confidentially accepted. Also, the T-values show that three cases of $\beta$-A $=2.5$ are characterized as "likely", while the most of them are characterized as "highly-likely".

Finally, in Figure 5, we plot the normalized RFU, averaged for all $\mathrm{M} \beta \mathrm{CD}$ (data from Table 4). We observe that the maximum of the normalized RFU occurs for $t \sim 1$ hour. We also show that the RFU differences between $\beta$-A $=0$ and $\beta$-A $=2.5$ is a constant value for any $t$, that is, $72 \pm 3$ (p-value $=0.49$; highly likely). This indicates that the response $\mathrm{RFU}(t)$ for various amounts of $\beta$-A differ mostly

Table 4. Normalized RFU constant values, with/without $\beta$-A, at each time $t$.

\begin{tabular}{|c|c|c|c|c|c|c|c|c|}
\hline \multicolumn{5}{|c|}{$\beta-A=0$} & \multicolumn{4}{|c|}{$\beta-\mathrm{A}=2.5$} \\
\hline$t[\mathrm{~min}]$ & RFU & $\chi_{\text {red }}^{2}$ & $p$-value & T-value & RFU & $\chi_{\text {red }}^{2}$ & $p$-value & T-value \\
\hline 15 & $111.5 \pm 0.6$ & 0.2 & 0.04 & -0.06 & $181 \pm 6$ & 0.62 & 0.32 & 0.75 \\
\hline 30 & $116.8 \pm 0.9$ & 0.55 & 0.26 & 0.64 & $192 \pm 8$ & 1.25 & 0.28 & 0.68 \\
\hline 45 & $122.6 \pm 0.8$ & 0.68 & 0.36 & 0.81 & $203 \pm 9$ & 1.6 & 0.15 & 0.39 \\
\hline 60 & $128.9 \pm 1.3$ & 0.57 & 0.27 & 0.66 & $213 \pm 11$ & 1.26 & 0.28 & 0.68 \\
\hline SS90 & $121.8 \pm 1.5$ & 0.52 & 0.24 & 0.60 & $204 \pm 8$ & 1.27 & 0.28 & 0.68 \\
\hline 120 & $108.8 \pm 1.3$ & 0.84 & 0.48 & 0.98 & $186 \pm 8$ & 1.85 & 0.1 & 0.23 \\
\hline 150 & $106.6 \pm 1.6$ & 0.84 & 0.48 & 0.98 & $180 \pm 7$ & 1.08 & 0.37 & 0.83 \\
\hline 180 & $102.1 \pm 1.2$ & 0.85 & 0.49 & 0.99 & $170 \pm 7$ & 1.35 & 0.24 & 0.60 \\
\hline 210 & $100 \pm 1.8$ & 1.13 & 0.34 & 0.78 & $165 \pm 6$ & 0.96 & 0.44 & 0.92 \\
\hline 240 & $110 \pm 2.1$ & 0.98 & 0.43 & 0.91 & $182 \pm 8$ & 1.31 & 0.26 & 0.64 \\
\hline 1060 & $95 \pm 3$ & 6.81 & $2.3 \mathrm{E}-06$ & -0.95 & $148 \pm 10$ & 2.08 & 0.06 & 0.06 \\
\hline
\end{tabular}


(a) p-values
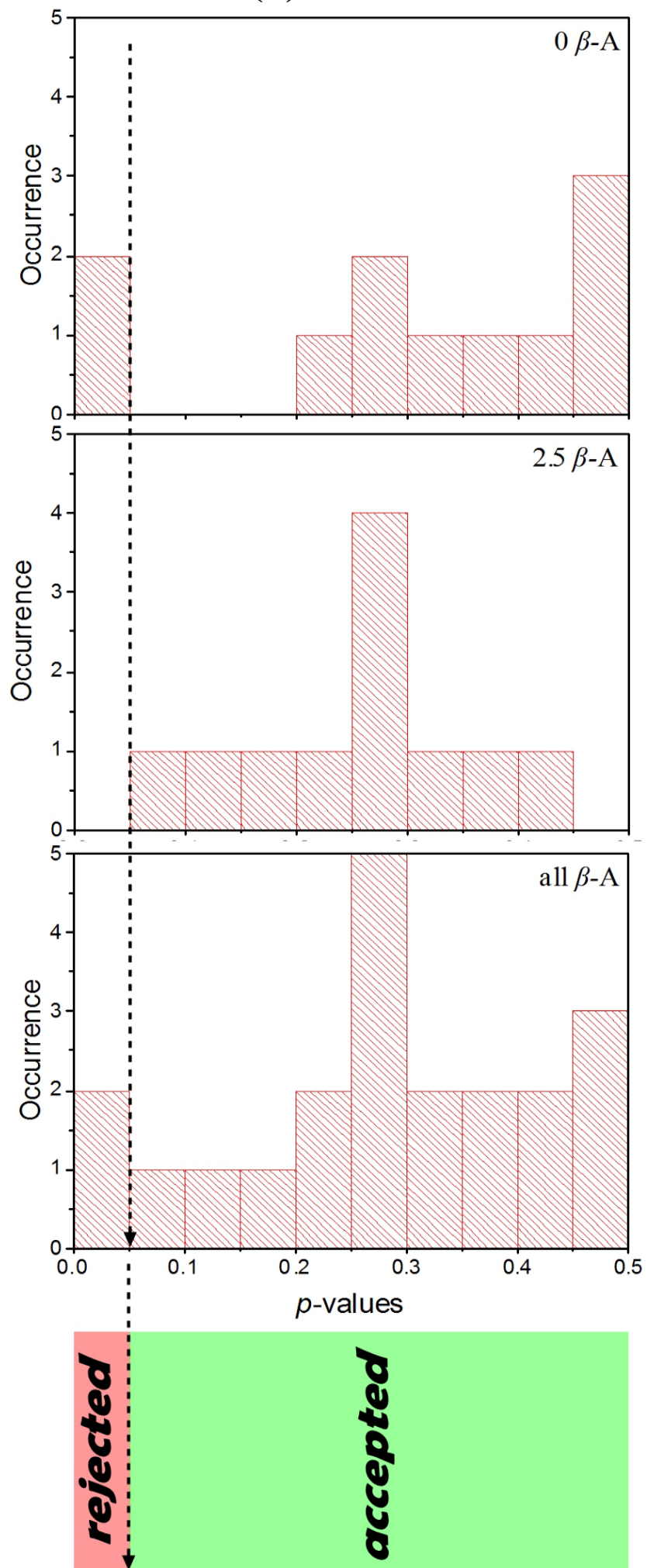

(b) $(T+1) / 2=(2 p)^{\log 2}$

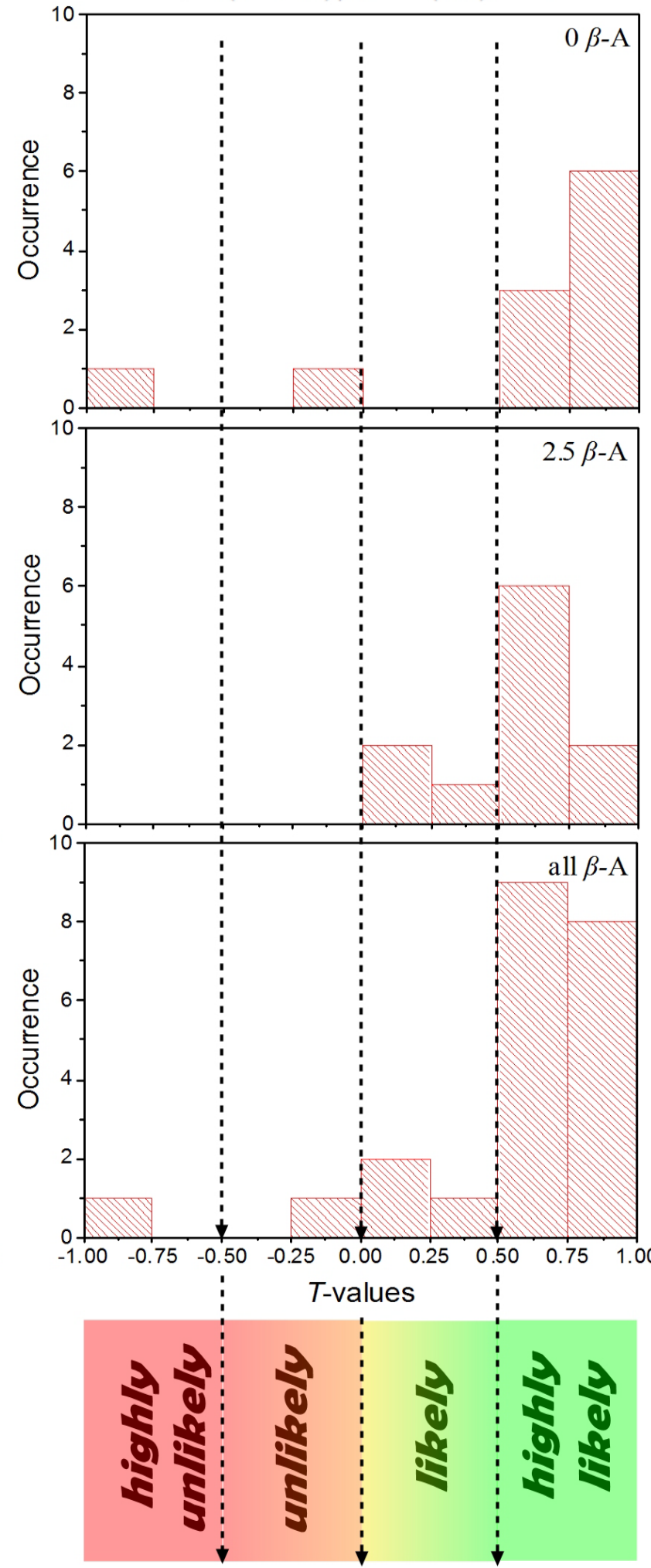

Figure 4. Characterization of the statistical hypothesis that the RFU values are invariant under variations of M $\beta C D$. (a) The histogram of the $p$-values show that all the cases of $\beta$-A $=2.5$ have the hypothesis accepted (rejected are the cases with $p$-values less than 0.05); (b) the histogram of the T-values show that the majority cases of $\beta$-A $=2.5$ are characterized with "highly likely". 


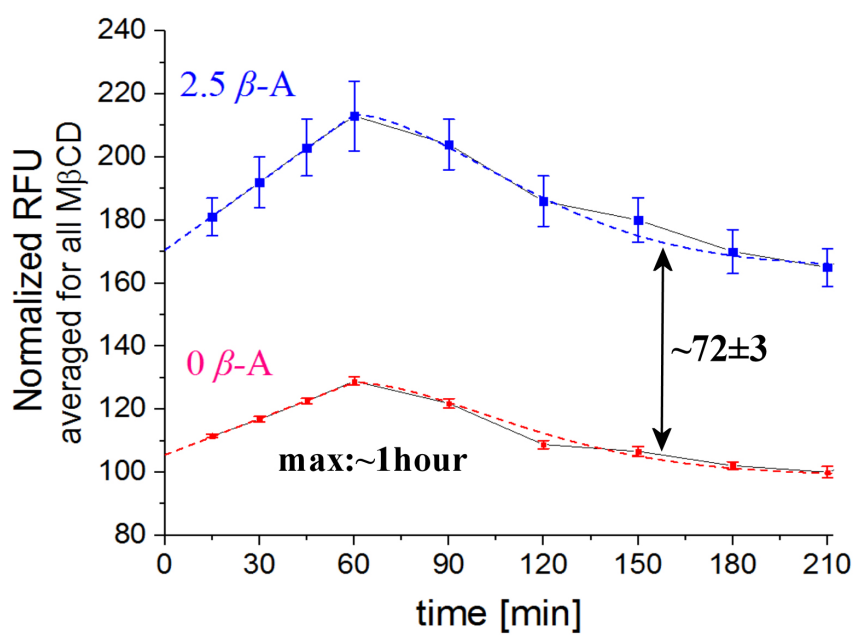

Figure 5. Characterization of the statistical hypothesis that the RFU values are invariant under variations of $\mathrm{M} \beta \mathrm{CD}$. (a) The histogram of the $p$-values show that all the cases of $\beta$-A $=2.5$ have the hypothesis accepted; (b) the histogram of the T-values show that the majority cases of $\beta-\mathrm{A}=2.5$ are characterized with "highly likely".

in the scaling. In other words, the graph of $\mathrm{RFU}(t)$ for $\beta-\mathrm{A}=2.5$ is a scaled replica of the graph of the graph of $\mathrm{RFU}(t)$ for $\beta$-A $=0$. Both the graphs appear to have linear behavior during the growth, while they decay exponential after the maximum RFU achieved for $t \sim 60$ minutes. Therefore, the graphs of RFU $(t)$ can be described by the same model, that is,

$$
\operatorname{RFU}(t)=\left\{\begin{array}{l}
\mathrm{RFU}_{0}+b_{\text {lin }} \cdot t, t \leq t_{\text {max }} \\
\mathrm{RFU}_{\infty}+b_{\text {exp }} \cdot e^{-\lambda \cdot\left|t-t_{\text {max }}\right|^{\gamma}}, t \geq t_{\text {max }}
\end{array},\right.
$$

Where $t_{\max }=60 \min , \lambda=0.00075, \gamma=1.7$ for both the cases of $\beta$-A $=0$ and $\beta-\mathrm{A}=$ 2.5; the values of $\mathrm{RFU}_{0}, b_{\text {lin }}, \mathrm{RFU}_{\infty}$, and $b_{\text {exp }}=\mathrm{RFU}_{\max }-\mathrm{RFU}_{\infty}$ are given by 105.5, $0.38,99,29.5$ and $170.5,0.72,165,48.5$, respectively for $\beta$ - $\mathrm{A}=0$ and $\beta-\mathrm{A}=2.5$.

\subsection{Experiment-3: Variation of $\beta$-A}

We observe that the addition of $\beta$-amyloid increased the mitochondrial activation. After $60 \mathrm{~min}$, the mitochondrial activation was at its peak, however it continued to fluctuate thereon after (Figure 6). Concentrations of beta amyloid 1 42 aggregates related directly with the increase in oxidative stress.

In order to demonstrate the independence of $\mathrm{RFU}(\beta-\mathrm{A})$ on $\mathrm{M} \beta \mathrm{CD}$, we proceed as follows: First, we derive the linear fitting of all the graphs of $\operatorname{RFU}(\beta-\mathrm{A})$, that is for the three different $\mathrm{M} \beta C \mathrm{CD}$ amounts $i=0,1,2.5 \mathrm{mM}$, and all the times $t$ $[\mathrm{min}]=0,15,30,45,60,90,120,150,180,210,240,270$ (Figure 7). The linear model $a+b \cdot[\beta-\mathrm{A}]$ fits the logarithm of the response, $\log (\mathrm{RFU})$, where we derive the optimal values of the fitting parameters $a$ and $b$. At each time $t$, we have three sets of derived values $a_{i} \pm \delta a_{i}$ and $b_{i} \pm \delta b_{p}$ one for each of the three different $\mathrm{M} \beta \mathrm{CD}$ concentrations, $i=0,1,2.5 \mathrm{mM}$.

Then, we average the three optimal values $a_{i}$ and $b_{p}$ for $i=1,2,3$. The averaged values $a \pm \delta a$ and $b \pm \delta b$, are shown in Table 5 and plotted in Figure 8(a), 


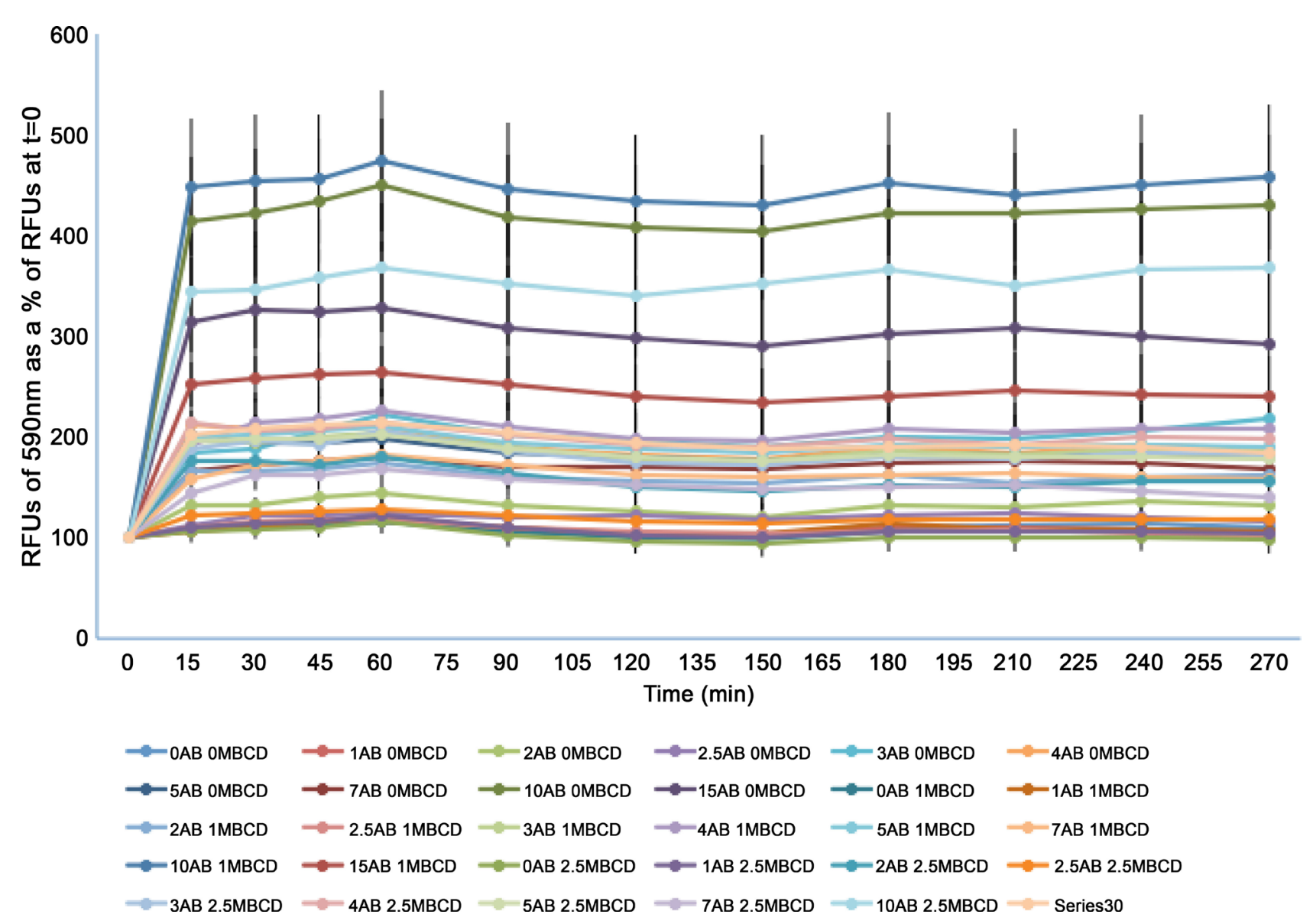

Figure 6. Mitochondrial activation of HT22 Hippocampal cells after beta amyloid and methyl- $\beta$-cyclodextrin in different amounts. Relative fluorescence unit at 590 of the fluorescence of mitochondrial stress because of the addition of mito-ID. For each set of $0,1,5 \mu \mathrm{M}$ of $\beta$-A and $\mathrm{M} \beta \mathrm{CD} n=12$ wells, and for each set of $\beta$-amyloid 2, 2.5, 3, 4, 7, 10, and $15 \mu \mathrm{M}$ of $\beta$ - $\mathrm{A}$ and $\mathrm{M} \beta \mathrm{CD} n=$ 6 wells for each set of $\beta$-A and $\mathrm{M} \beta \mathrm{CD}$.
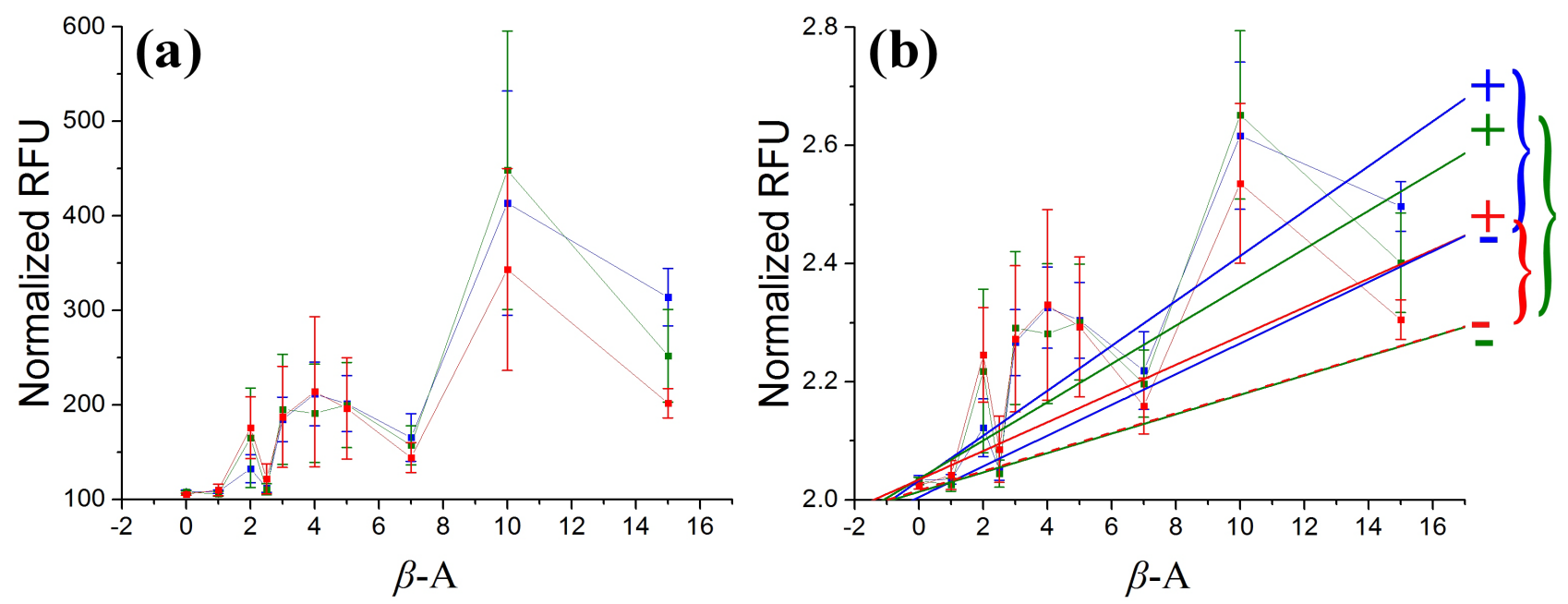

Figure 7. (a) Dose response RFU at $t=15 \mathrm{~min}$ for the mitochondrial activation of HT22 hippocampal cells with $0 \mathrm{mM}$ (blue), 1 $\mathrm{mM}$ (green), and $2.5 \mathrm{mM}$ (red)of $\mathrm{M} \beta \mathrm{CD}$, and various $\beta$-amyloid concentrations $[\mu \mathrm{M}]$. (b) The same plot but the RFU is depicted on a $\log$ scale, where we co-plot the linear fits $y=(a \pm \delta a)+(b \pm \delta b) \cdot x$. In particular, we have applied a linear fitting $y_{j}=a+b \cdot x_{j}$ where $y \equiv \log (\mathrm{RFU})$ and $x \equiv[\beta-\mathrm{A}]$, from which we find $a \pm \delta a$ and $b \pm \delta b$, and co-plot the cases of $y=(a+\delta a)+(b+\delta b) \cdot x$ (case “+”) and $y=(a-\delta a)+(b-\delta b) \cdot x$ (case “-”). 
Table 5. Averaged fitting parameters $a$ and $b$.

\begin{tabular}{ccccccc}
\hline \multicolumn{5}{c}{$\beta$-A $=0$} & & \multicolumn{3}{c}{$\beta$-A $=2.5$} \\
\hline [min] & $a$ & $p$-value & T-value & $b$ & $p$-value & T-value \\
\hline 15 & $2.014 \pm 0.007$ & 0.08 & 0.14 & $0.024 \pm 0.005$ & 0.27 & 0.66 \\
30 & $2.038 \pm 0.008$ & 0.28 & 0.68 & $0.024 \pm 0.004$ & 0.44 & 0.93 \\
45 & $2.047 \pm 0.009$ & 0.22 & 0.55 & $0.024 \pm 0.005$ & 0.24 & 0.59 \\
60 & $2.061 \pm 0.014$ & 0.20 & 0.51 & $0.023 \pm 0.005$ & 0.22 & 0.57 \\
90 & $2.018 \pm 0.008$ & 0.11 & 0.28 & $0.030 \pm 0.004$ & 0.46 & 0.95 \\
120 & $2.002 \pm 0.011$ & 0.21 & 0.53 & $0.031 \pm 0.003$ & 0.41 & 0.88 \\
150 & $1.994 \pm 0.012$ & 0.31 & 0.74 & $0.0309 \pm 0.0029$ & 0.36 & 0.81 \\
180 & $2.023 \pm 0.014$ & 0.47 & 0.97 & $0.030 \pm 0.003$ & 0.27 & 0.66 \\
210 & $2.025 \pm 0.014$ & 0.32 & 0.75 & $0.030 \pm 0.003$ & 0.18 & 0.48 \\
240 & $2.026 \pm 0.019$ & 0.40 & 0.87 & $0.029 \pm 0.003$ & 0.22 & 0.56 \\
270 & $2.017 \pm 0.022$ & 0.38 & 0.85 & $0.029 \pm 0.003$ & 0.25 & 0.62 \\
\hline
\end{tabular}

Notes. Fitting parameters $a$ and $b$, averaged over their three values $a_{i}$ and $b_{i}$ derived for three different $\mathrm{M} \beta \mathrm{CD}$ amounts $i=0,1,2.5 \mathrm{mM}$, all at each time $t$, with the statistical $p$-values and T-values of each averaging also shown.

Figure 8(b) with respect to $t$. For each averaging, we state the following statistical hypothesis:

- $\mathrm{H}_{0}$ : the three linear fits are the same, namely, $a_{1}=a_{2}=a_{3}$ and $b_{1}=b_{2}=b_{3}$.

For this hypothesis to be accepted or rejected is necessary to check the $p$-values of the fitting, as in Experiment-2. The estimated $p$-values, as well as the derived T-values, are also shown in Table 5 and plotted in Figures 8(c)-(f) with respect to $t$.

We observe that all the $\mathrm{p}$-values approve the statistical hypothesis that the linear behavior of $\log (\mathrm{RFU})$ with $\beta$ - $\mathrm{A}$ is independent of the amount of the $\mathrm{M} \beta \mathrm{CD}$, for all the times of the experiment. In particular, all the $\mathrm{p}$-values are larger of the confident level of 0.05 (Figure 8(c), Figure 8(d)). They all correspond to large T-values, which are characterized as highly likely, except of three cases (two for the intercept $a$ and one for the slope $a$ ) characterized as likely (Figure 8(e), Figure $8(f))$. Therefore, the results do not show any differences with changes in cholesterol content of the plasma membrane.

\section{Discussion and Conclusions}

Data for three types of experiments, as regards the influence of cholesterol on the $\beta$-Amyloid, were collected and statistically analyzed. The three experiments are the following: 1) Correspondence between cholesterol and methyl- $\beta$-cyclodextrin $(\mathrm{M} \beta \mathrm{CD})$ measurements; 2) measurements of the relative fluorescence unit (RFU) with respect to $\mathrm{M} \beta \mathrm{CD}$ concentration (with/without $\beta$-A); and 3) RFU measurements with respect to $\beta$-A concentration (with/without $\mathrm{M} \beta \mathrm{CD}$ ). The statistical analysis of the presented experimental datasets affirms that cholesterol 

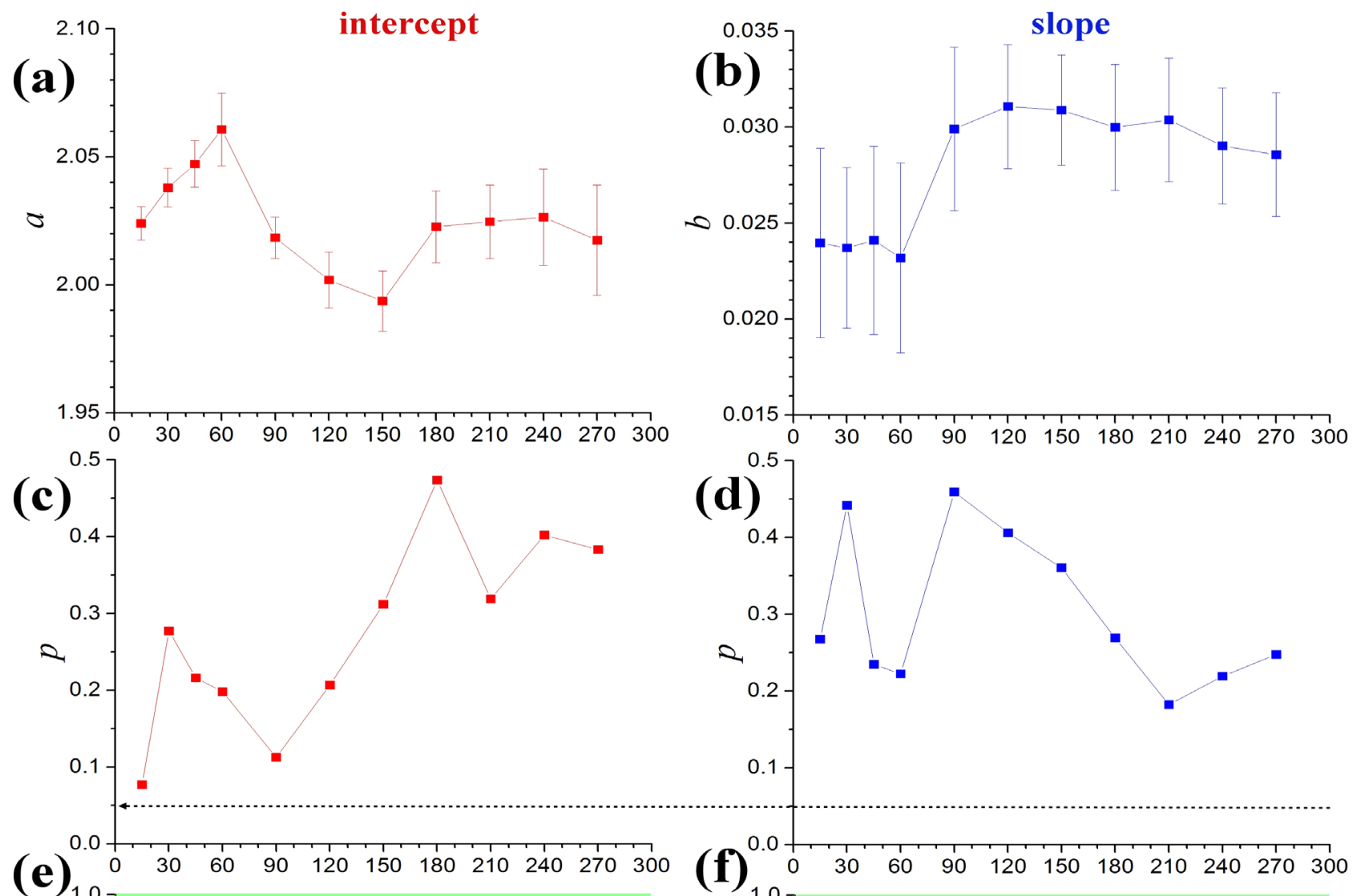

(d)
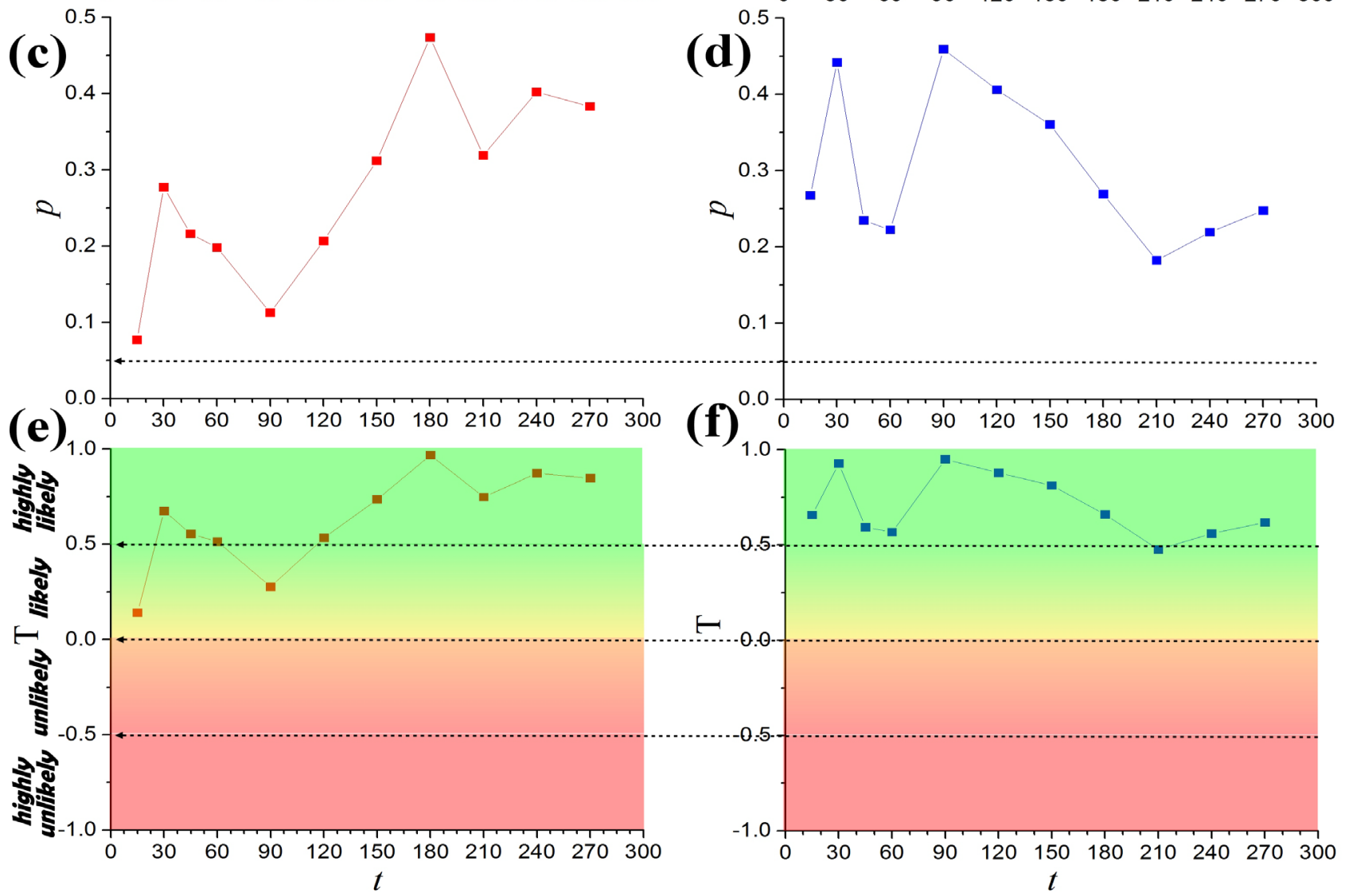

Figure 8. The average values of the (a) intercept $a$, and (b) slope $b$, are plotted with respect to time $t$. The statistical $p$-values and T-values of averaging $a_{i}$ and $b_{i}$ (derived for three different $\mathrm{M} \beta \mathrm{CD}$ amounts $i=0,1,2.5 \mathrm{mM}$ ), are plotted in ((c), (e)) and ((d), (f)), respectively.

has no statistically significant effect on the degree of toxicity of $\beta$-Amyloid. This also confirms the conclusion of Dayeh et al. [37], where the authors showed that there is no significant effect of altering cholesterol on cell toxicity by hydrogen peroxide.

We did observe an increase in mitochondrial activation with increasing concentrations of $\beta$ - $\mathrm{A}$ as evidenced by the data in Figure 7. The increase in mitochondrial activation is an indicator for the oxidative stress the cells experienced. Thus, the addition of small oligomer $\beta$-A does seem to induce some stress re- 
sponse in HT22 cells. The drop in response at $7 \mu \mathrm{M}$ and $15 \mu \mathrm{M}$ may be due to nonspecific responses to the $\beta$-A peptide. We did not, however, observe statistically significant differences with changes in cholesterol content of the plasma membrane (Figure 8). Treatment of the cells with $1 \mathrm{mM} \mathrm{M} \beta \mathrm{CD}$ resulted in a reduction of $44 \%$ of the overall cholesterol content of the cells, while $2.5 \mathrm{mM}$ $\mathrm{M} \beta \mathrm{CD}$ resulted in a $74 \%$ decrease. The plasma membrane contains approximately half of the cholesterol in the cell, which means that at $74 \%$ decrease in total cholesterol is a theoretical $37 \%$ decrease in the plasma membrane alone. We feel that this is a significant portion of the cholesterol and that this decrease would lead to alterations of lipid raft structures.

Over time, we did not see a statistically significant decrease in mitochondrial activity due to $\beta$-A's toxicity. The negative control also had fluctuations in its mitochondrial activity over time. This lack of collapse in the mitochondrial activation indicated that the cells did not apoptose. We used HT22 hippocampal cells that are treated with a simian virus making them tumor cells. Since these hippocampal cells replicate, the effects of $\beta$-A can be compromised with the assay we used. The Mito-ID only provides information about the general population of cells. Due to heterogenous responses among HT22 cells, the assay was not sensitive enough to detect cellular-level differences in response to $\beta$-A and changes in cholesterol content. The cells also secrete growth factors that have protected them from the oxidative stress.

Cholesterol has many important roles in cellular function. It provides structure for the neuronal membrane, and plays an important part in cellular signaling. Our initial hypothesis is that cholesterol in the lipid membrane would play a protective role against oxidative stress. With this particular model, cholesterol content in the lipid layer does not seem to play any detectable role in modulating the effects of beta amyloid on oxidative stress response.

- We observe an increase in mitochondrial activation with increasing concentrations of beta amyloid. Dose response shows increasing relative mitochondrial activation.

- We did not see statistically significant differences with changes in cholesterol content of the plasma membrane. There are no statistically significant differences in mitochondrial activation at the same beta amyloid concentration between cells with different cholesterol contents.

- We did check that the cholesterol content of the membrane decreased with increasing treatment concentrations of $\mathrm{M} \beta \mathrm{CD}$. The plasma membrane contains approximately $50 \%$ of the cell's cholesterol, so a reduction of $50 \%$ or $75 \%$ of the overall cholesterol significantly reduces plasma membrane cholesterol content.

- We observed lack of collapse of mitochondrial potential over time. Possibly, the dividing cells protect themselves against beta amyloid.

- Cholesterol content does not seem to play a significant role in modulating the effects of beta amyloid on oxidative stress response. 
- HT22 neurons treated with human $\beta$-amyloid $(1-40)$ showed oxidative stress.

We conclude with what's next for improving and extending the presented analysis. Other investigations may involve other effects of membrane cholesterol changes. For example, Nicholson and collaborators suggested that age-dependent changes in membrane cholesterol might, at least in part, modulate the susceptibility of hippocampal neurons to $\mathrm{A} \beta$-induced $\mathrm{Ca}^{2+}$ influx, calpain activation, and subsequent Tau toxicity in an NMDA receptor-dependent manner [26]. Other methods cantest different oligomers preparations (small or high molar weight oligomers up to fibrils) or the effect of different amyloid peptides (e.g., amyloid-beta40, amyloid-beta42). Moreover, differentiated HT22 neurons represent a better model of hippocampal neurons than undifferentiated cells (e.g., [38]). Mature neurons are known to be post-mitotic cells and have distinct cellular properties as opposed to their mitotic precursor cells. Sensitivity of HT22 cells to toxicity inducers changes dramatically when comparing undifferentiated with differentiated cells. Differentiation is important for immortalized cell lines to render post-mitotic neuronal properties. Then, experimentation with differentiated HT22 neurons (as described in [39]) can exclude the possibility that the results reflect mostly the cellular properties of immature hippocampal neuronal precursor cells rather than post-mitotic hippocampal neurons. In addition, lipids regulate the beta-secretase beta-site APP cleavage enzyme 1 (BACE1). There is a cholesterol-dependent activity of gamma-secretase which links the interrelationship between a cholesterol-enriched lipid raft and amyloidogenic processing of APP. Future work may also determine BACE1 and gamma-secretase activities along with mitochondrial membrane potential (e.g., [40]), in order to display a better picture of the cytotoxicity event.

\section{Acknowledgements}

This work was supported by a grant from King Abdul Aziz University, S. A.

\section{References}

[1] Edland, S.D., Slager, S. and Farrer, M. (2004) Genetic Association Studies in Alzheimer's Disease Research: Challenges and Opportunities. Statistics in Medicine, 23, 169-178. https://doi.org/10.1002/sim.1706

[2] Pollard, H.B., Arispe, N. and Rojas, E. (1995) Ion Channel Hypothesis for Alzheimer Amyloid Peptide Neurotoxicity. Cellular and Molecular Neurobiology, 15, 513-526. https://doi.org/10.1007/BF02071314

[3] Butterfield, S.M. and Lashuel, H.A. (2010) Amyloidogenic Protein-Membrane Interactions: Mechanistic Insight from Model Systems. Angewandte Chemie International Edition, 49, 5628-5654. https://doi.org/10.1002/anie.200906670

[4] Williams, T.L. and Serpell, L.C. (2011) Membrane and Surface Interactions of Alzheimer's $\beta$-A Peptide-Insights into the Mechanism of Cytotoxicity. FEBS Journal, 278, 3905-3917. https://doi.org/10.1111/j.1742-4658.2011.08228.x

[5] Quist, A., Doudevski, I., Lin, H., Azimova, R., Ng, D., Frangione, B., Kagan, B., Ghiso, J. and Lal, R. (2005) Amyloid Ion Channels: A Common Structural Link for 
Protein-Misfolding Disease. PNAS, 102, 10427-10432.

https://doi.org/10.1073/pnas.0502066102

[6] Connelly, L., Arc, F.T., Jang, H., Capone, R., Kotler, S., Ramachsaandran, S., Kagan, B., Nussinov, R. and Lal, R. (2012) Atomic Force Microscopy and MD Simulations Reveal Pore-Like Structures of all-D-enantiomer of Alzheimer's $\beta$-Amyloid Peptide: Relevance to the Ion Channel Mechanism of AD Pathology. The Journal of Physical Chemistry B, 116, 1728-1735. https://doi.org/10.1021/jp2108126

[7] Kayed, R., Head, E., Thompson, J.L., McIntire, T.M., Milton, S.C., Cotman, C.W. and Glabe, C.G. (2003) Common Structure of Soluble Amyloid Oligomers Implies Common Mechanism of Pathogenesis. Science, 300, 486-489.

https://doi.org/10.1126/science.1079469

[8] Demuro, A., Mina, E., Kayed, R., Milton, S.C., Parker, I. and Glabe, C.G. (2005) Calcium Dysregulation and Membrane Disruption as a Ubiquitous Neurotoxic Mechanism of Soluble Amyloid Oligomers. The Journal of Biological Chemistry, 280, 17294-17300. https://doi.org/10.1074/jbc.M500997200

[9] Van Meer, G., Voelker, D.R. and Feigenson, G.W. (2008) Membrane Lipids: Where They Are and How They Behave. Nature Reviews Molecular Cell Biology, 9, 112-124. https://doi.org/10.1038/nrm2330

[10] Simons, M., Keller, P., Destrooper, B., Beyreuther, K., Dotti, C.G. and Simons, K. (1998) Cholesterol Depletion Inhibits the Generation of Beta-Amyloid in Hippocampal Neurons. PNAS, 95, 6460-6464. https://doi.org/10.1073/pnas.95.11.6460

[11] Rushworth, J.V. and Hooper, N.M. (2011) Lipid Rafts: Linking Alzheimer's Amyloid- $\beta$ Production, Aggregation, and Toxicity at Neuronal Membranes. International Journal of Alzheimer's Disease, 2011, Article ID: 603052.

[12] Sorensen, A.A. and Weedon, D. (2016) The Top 100 Cited Cholesterol Papers. Biomedical Spectroscopy and Imaging, 5, S5-S43. https://doi.org/10.3233/BSI-160160

[13] Vance, J.E., Hayashi, H. and Karen, B. (2005) Cholesterol Homeostasis in Neurons and Glial Cells. Seminars in Cell \& Developmental Biology, 16, 193-212. https://doi.org/10.1016/j.semcdb.2005.01.005

[14] Romano, T.A., Ridgway, S.H., Felten, D.L. and Quaranta, V. (1999) Molecular Cloning and Characterization of CD4 in an Aquatic Mammal, the White Whale Delphinapterus leucas. Immunogenetics, 49, 376-383. https://doi.org/10.1007/s002510050510

[15] Jarvik, G.P. (1998) Complex Segregation Analyses: Uses and Limitations. The American Journal of Human Genetics, 63, 942-946. https://doi.org/10.1086/302075

[16] Evans, R.M., Emsley, C.L., Gao, S., Sahota, A., Hall, K.S., Farlow, M.R. and Hendrie, H. (2000) Serum Cholesterol, APOE Genotype, and the Risk of Alzheimer's Disease: A Population-Based Study of African Americans. Neurology, 54, 240-242. https://doi.org/10.1212/WNL.54.1.240

[17] Kivipelto, M., Laasko, M.P., Tuomilehto, J., Nissinen, A. and Soininen, H. (2002) Hypertension and Hypercholesterolaemia as Risk Factors for Alzheimer's Disease: Potential for Pharmacological Intervention. CNS Drugs, 16, 435-444. https://doi.org/10.2165/00023210-200216070-00001

[18] Anstey, K.J., Ashby-Mitchell, K. and Peters, R. (2017) Updating the Evidence on the Association between Serum Cholesterol and Risk of Late-Life Dementia: Review and Meta-Analysis. Journal of Alzheimer's Disease, 56, 215-228.

https://doi.org/10.3233/JAD-160826 
[19] Arispe, N., Pollard, H.B. and Rojas, E. (1994) Beta-Amyloid Ca(2+)-channel Hypothesis for Neuronal Death in Alzheimer Disease. Molecular and Cellular Biochemistry, 140, 119-125. https://doi.org/10.1007/BF00926750

[20] Cecchi, C., Nichino, D., Zampagni, M., Bernacchioni, C., Evangelisti, E., Pensalfini, A., Liguri, G., Gliozzi, A., Stefani, M. and Relini, A. (2009) A Protective Role for Lipid Raft Cholesterol against Amyloid-Induced Membrane Damage in Human Neuroblastoma Cells. Biochimica et Biophysica Acta, 1788, 2204-2216.

[21] Schneider, A., Schulz-Schaeffer, W., Hartmann, T., Schulz, J.B. and Simons, M. (2006) Cholesterol Depletion Reduces Aggregation of Amyloid-Beta Peptide in Hippocampal Neurons. Neurobiology of Disease, 23, 573-577. https://doi.org/10.1016/j.nbd.2006.04.015

[22] De Chaves, E. (2012) Lipids in Lipids in Alzheimer's Disease: A New Link between Cholesterol and a Beta Toxicity. Canadian Journal of Physiology and Pharmacology, 90, 674-675.

[23] Uranga, R.M. and Keller, J.N. (2010) Diet and Age Interactions with Regards to Cholesterol Regulation and Brain Pathogenesis. Current Gerontology and Geriatrics Research, 2010, Article ID: 219683. https://doi.org/10.1155/2010/219683

[24] Brosche, T. and Platt, D. (1990) Decrease of Cholesterol Concentration in Human Erythrocyte Ghosts in Old Age. Experimental Gerontology, 25, 23-28. https://doi.org/10.1016/0531-5565(90)90005-M

[25] Zhang, J. and Liu, Q. (2015) Cholesterol Metabolism and Homeostasis in the Brain. Protein Cell, 6, 254-264. https://doi.org/10.1007/s13238-014-0131-3

[26] Nicholson, A.M. and Ferreira, A. (2009) Increased Membrane Cholesterol Might Render Mature Hippocampal Neurons More Susceptible to Beta-Amyloid-Induced Calpain Activation and Tau Toxicity. Journal of Neuroscience, 29, 4640-4651. https://doi.org/10.1523/JNEUROSCI.0862-09.2009

[27] Nicholson, A.M., Methner, D.N.R. and Ferreira, A. (2011) Membrane Cholesterol Modulates $\beta$-Amyloid-Dependent Tau Cleavage by Inducing Changes in the Membrane Content and Localization of N-Methyl-d-Aspartic Acid Receptors. The Journal of Biological Chemistry, 286, 976-986. https://doi.org/10.1074/jbc.M110.154138

[28] McKay, R. and DiMaio, D. (1981) Binding of an SV40 T Antigen-Related Protein to the DNA of SV40 Regulatory Mutants. Nature, 289, 810-813.

https://doi.org/10.1038/289810a0

[29] Woollacott, A.J. and Simpson, P.B. (2001) High Throughput Fluorescence Assays for the Measurement of Mitochondrial Activity in Intact Human Neuroblastoma Cells. Journal of Biomolecular Screening, 6, 413-420. https://doi.org/10.1177/108705710100600607

[30] Livadiotis, G. (2007) Approach to General Methods for Fitting and Their Sensitivity. Physica A, 375, 518-536. https://doi.org/10.1016/j.physa.2006.09.027

[31] Livadiotis, G. (2014) Chi-p Distribution: Characterization of the Goodness of the Fitting using Lp Norms. Journal of Statistical Distributions and Applications, 1, 4. https://doi.org/10.1186/2195-5832-1-4

[32] Frisch, P.C., Bzowski, M., Livadiotis, G., McComas, D.J., Möbius, E., Mueller, H.-R., Pryor, W.R., Schwadron, N.A., Sokól, J.M., Vallerga, J.V. and Ajello, J.M. (2013) Decades-Long Changes of the Interstellar Wind through Our Solar System. Science, 341, 1080. https://doi.org/10.1126/science.1239925

[33] Livadiotis, G. and McComas, D.J. (2013) Evidence of Large Scale Phase Space Quantization in Plasmas. Entropy, 15, 1118-1132.

https://doi.org/10.3390/e15031118 
[34] Schwadron, N.A., Mobius, E., Kucharek, H., Lee, M.A., French, J., Saul, L., Wurz, P., Bzowski, M., Fuselier, S., Livadiotis, G., McComas, D.J., Frisch, P., Gruntman, M. and Mueller, H. (2013) Solar Radiation Pressure and Local Interstellar Medium Flow Parameters from IBEX Low Energy Hydrogen Measurements. The Astrophysical Journal, 775, 86. https://doi.org/10.1088/0004-637X/775/2/86

[35] Fuselier, S.A., Allegrini, F., Bzowski, M., Dayeh, M.A., Desai, M., Funsten, H.O., Galli, A., Heirtzler, D., Janzen, P., Kubiak, M.A., Kucharek, H., Lewis, W., Livadiotis, G., McComas, D.J., Möbius, E., Petrinec, S.M., Quinn, M., Schwadron, N., Sokół, J.M., Trattner, K.J., Wood, B.E. and Wurz, P. (2014) Low Energy Neutral Atoms from the Heliosheath. The Astrophysical Journal, 784, 89.

https://doi.org/10.1088/0004-637X/784/2/89

[36] Livadiotis, G. and Moussas, X. (2007) The Sunspot as an Autonomous Dynamical System: A Model for the Growth and Decay Phases of Sunspots. Physica A, 379, 436-458. https://doi.org/10.1016/j.physa.2007.02.003

[37] Dayeh, M., Assas, L., Aminian, F., Cheng, K.M., Elaydi, S., Livadiotis, G., Moreno, V., Viswasam, N. and Roberts, J.L. (2017) The Role of Cholesterol in Stress Related Neuronal Death.

[38] He, M., Liu, J., Cheng, S., Xing, Y. and Suo, W.Z. (2013) Differentiation Renders Susceptibility to Excitotoxicity in HT22 Neurons. Neural Regeneration Research, 8 , 1297-1306.

[39] Suo, Z., Wu, M., Citron, B.A., et al. (2003) Rapid Tau Aggregation and Delayed Hippocampal Neuronal Death Induced by Persistent Thrombin Signaling. The Journal of Biological Chemistry, 278, 37681-37689. https://doi.org/10.1074/jbc.M301406200

[40] Schaefer, P.M., von Einem, B., Walther, P., Calzia, E. and von Arnim, C.A.F. (2016) Metabolic Characterization of Intact Cells Reveals Intracellular Amyloid Beta but Not Its Precursor Protein to Reduce Mitochondrial Respiration, PLoS ONE, 11, e0168157. https://doi.org/10.1371/journal.pone.0168157 